ARTICLE OPEN

\title{
Osteogenesis imperfecta mutations in plastin 3 lead to impaired calcium regulation of actin bundling
}

\author{
Christopher L. Schwebach $\mathbb{D}^{1,2}$, Elena Kudryashova $\mathbb{D}^{1}$, Weili Zheng $\mathbb{D}^{3}$, Matthew Orchard ${ }^{1}$, Harper Smith ${ }^{1,4}$, Lucas A. Runyan ${ }^{1}$, \\ Edward H. Egelman (iD ${ }^{3}$ and Dmitri S. Kudryashov (iD) ${ }^{1,2,4}$
}

\begin{abstract}
Mutations in actin-bundling protein plastin 3 (PLS3) emerged as a cause of congenital osteoporosis, but neither the role of PLS3 in bone development nor the mechanisms underlying PLS3-dependent osteoporosis are understood. Of the over 20 identified osteoporosis-linked PLS3 mutations, we investigated all five that are expected to produce full-length protein. One of the mutations distorted an actin-binding loop in the second actin-binding domain of PLS3 and abolished F-actin bundling as revealed by cryo-EM reconstruction and protein interaction assays. Surprisingly, the remaining four mutants fully retained F-actin bundling ability. However, they displayed defects in $\mathrm{Ca}^{2+}$ sensitivity: two of the mutants lost the ability to be inhibited by $\mathrm{Ca}^{2+}$, while the other two became hypersensitive to $\mathrm{Ca}^{2+}$. Each group of the mutants with similar biochemical properties showed highly characteristic cellular behavior. Wild-type PLS3 was distributed between lamellipodia and focal adhesions. In striking contrast, the $\mathrm{Ca}^{2+}$-hyposensitive mutants were not found at the leading edge but localized exclusively at focal adhesions/stress fibers, which displayed reinforced morphology. Consistently, the $\mathrm{Ca}^{2+}$-hypersensitive PLS3 mutants were restricted to lamellipodia, while chelation of $\mathrm{Ca}^{2+}$ caused their redistribution to focal adhesions. Finally, the bundling-deficient mutant failed to co-localize with any F-actin structures in cells despite a preserved F-actin binding through a non-mutation-bearing actin-binding domain. Our findings revealed that severe osteoporosis can be caused by a mutational disruption of the $\mathrm{Ca}^{2+}$-controlled PLS3's cycling between adhesion complexes and the leading edge. Integration of the structural, biochemical, and cell biology insights enabled us to propose a molecular mechanism of plastin activity regulation by $\mathrm{Ca}^{2+}$.
\end{abstract}

Bone Research (2020)8:21

; https://doi.org/10.1038/s41413-020-0095-2

\section{INTRODUCTION}

Osteoporosis is a disease defined by low bone density and disruption of the bone architecture resulting in fragility and fractures. ${ }^{1}$ Hereditary forms of bone fragility called osteogenesis imperfecta (OI) or "brittle bone disease" are mostly linked to dysregulation of Type I collagen. ${ }^{2}$ Approximately $90 \%$ of OI cases stem from mutations in collagen I genes, ${ }^{3,4}$ while most of the remaining forms affect collagen-processing enzymes involved in collagen folding, posttranslational modifications, intracellular transport, or matrix incorporation. ${ }^{4}$ Recently, several cases of OI with classical clinical manifestations in hemizygous men and a variable phenotype in heterozygous women, but without an obvious link to collagen, were attributed to mutations in an Xchromosome gene coding an actin-bundling protein plastin 3 (PLS3). ${ }^{5-17}$

Among three vertebrate tissue-specific plastin isoforms, ${ }^{18}$ PLS3 (also known as T-plastin) is ubiquitously expressed in solid tissues $^{19}$ and involved in cell migration, ${ }^{20}$ endocytosis, ${ }^{21}$ DNA repair, $^{22}$ and membrane trafficking. ${ }^{23}$ In agreement with the essential role of PLS3 in bone and connective tissue development in vertebrates, a pls3 knockdown in zebrafish results in craniofacial dysplasia and malformations of body axis and tail, ${ }^{13}$ whereas PLS3 knockout mouse models showed impaired cortical bone acquisition with decreased osteoblast mineralization capacity ${ }^{24}$ and defects in the development of the epidermal basal membrane. ${ }^{25}$ In humans, PLS3 mutations were also associated with a diaphragmatic hernia. ${ }^{26}$ However, a detailed understanding of PLS3's contribution to any of the above-mentioned cellular processes or to osteogenesis is missing.

The domain structure of PLS3 (Fig. 1a) encompasses the Nterminal $\mathrm{Ca}^{2+}$-binding regulatory domain (RD) and a core consisting of two actin-binding domains (ABD1 and ABD2). RD contains two EF-hands and a calmodulin-binding motif (CBM), whereas each $A B D$ is assembled from two tandem calponinhomology $(\mathrm{t}-\mathrm{CH})$ domains. Binding of $\mathrm{Ca}^{2+}$ ions by EF-hands potently inhibits F-actin bundling, but has only a marginal effect on F-actin binding by all human isoforms, ${ }^{27}$ suggesting that only one of the ABD's binding to actin is inhibited. RD is connected to the $A B D$ core via a linker (Fig. 1a), whose length and likely flexibility precluded, thus far, mapping of the RD's place in the tertiary structure of plastins; hence, the mechanism of the $\mathrm{Ca}^{2+}$-dependent regulation remains unknown.

Of over 20 Ol-linked mutations of PLS3 identified to date in osteoporosis patients, ${ }^{5-17}$ five are insertions or missense

\footnotetext{
'Department of Chemistry and Biochemistry, The Ohio State University, Columbus, OH 43210, USA; ${ }^{2}$ Molecular Cellular and Developmental Biology graduate program, The Ohio State University, Columbus, OH 43210, USA; ${ }^{3}$ Department of Biochemistry and Molecular Genetics, University of Virginia, Charlottesville, VA 22908 , USA and ${ }^{4}$ Biophysics graduate program, The Ohio State University, Columbus, $\mathrm{OH} 43210$, USA

Correspondence: Dmitri S. Kudryashov (kudryashov.1@osu.edu)

These authors contributed equally: Christopher L. Schwebach, Elena Kudryashova
}

Received: 2 September 2019 Revised: 6 February 2020 Accepted: 23 March 2020

Published online: 22 May 2020 


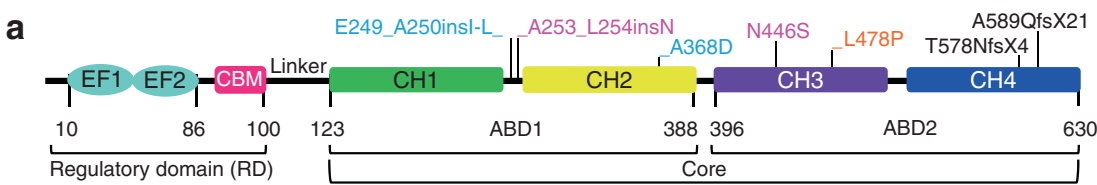

b

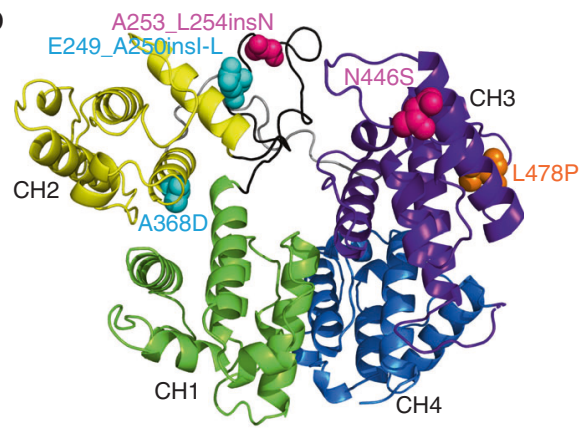

d

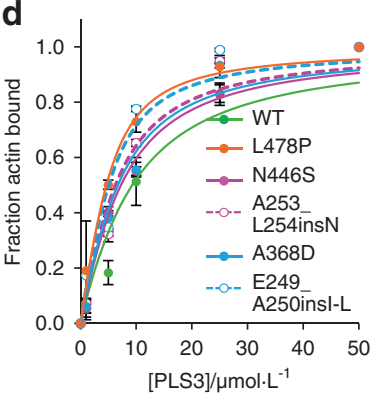

e

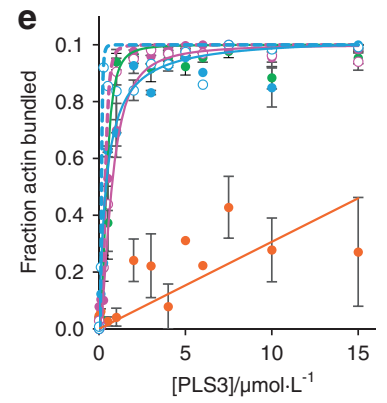

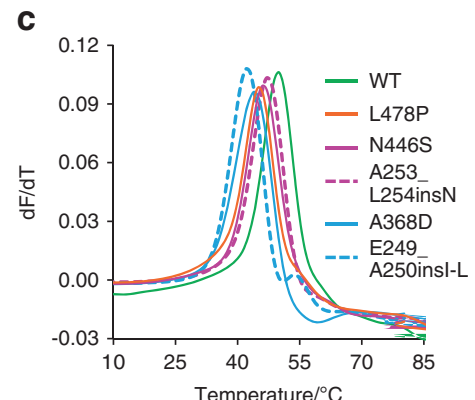

f

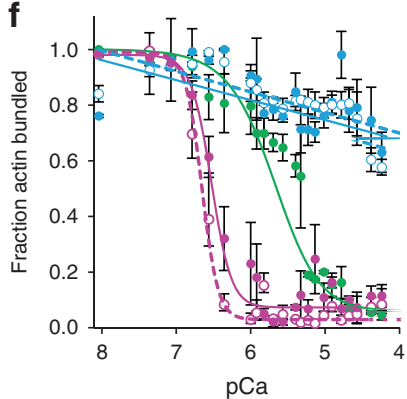

Fig. 1 PLS3 domain structure and effects of OI-linked PLS3 mutations on PLS3 properties (see also Supplementary Fig. S1). a A schematic diagram of plastin domain structure: EF EF-hands motifs, CBM calmodulin-binding motif, RD N-terminal regulatory domain, $\mathrm{CH}$ calponinhomology domains, ABD actin-binding domains, Core actin-binding core domain, Linker a flexible linker separating the CBM and ABD1. PLS3 amino acid residue numbers and the OI-causative PLS3 mutations are shown below and above the diagram, respectively. $\mathbf{b}$ A homologybased model of the PLS3 actin-binding core (color scheme as in a) generated by Phyre2. ${ }^{86}$ c Melting profiles of PLS3 osteoporosis mutants were recorded by DSF in three independent repetitions; averaged data were plotted as the negative first derivatives of fluorescence signals versus temperature with transition temperatures $\left(T_{m}\right)$ summarized in Table 1. F-actin binding (d) and bundling (e) by WT and mutated PLS3 were analyzed by high- and low-speed co-sedimentation and characterized by $\mathrm{K}_{d}$ and [PLS3 $]_{50 \%}$ respectively (given in Table 1). Error bars represent standard errors of the mean of three and two independent repetitions for binding and bundling experiments, respectively (see also Supplementary Fig. S4). f Sensitivity of the PLS3 mutants to $\mathrm{Ca}^{2+}$ was measured by the decrease in light scattering reflecting the dissociation of plastin-mediated F-actin bundles upon titration with $\mathrm{CaCl}_{2}$. Error bars represent standard errors of the mean of three independent experiments. The data were fit to a logistic curve to determine the $\mathrm{pCa}_{50 \%}$ (provided in Table 1)

mutations that are predicted to produce full-length protein, while the remaining are either gene deletions or nonsense/frameshift mutations expected, and in some cases demonstrated, ${ }^{13,16}$ to produce no protein owing to a nonsense-mediated mRNA decay. ${ }^{28,29}$ Of the former five (Fig. 1a, b), a 12-amino-acid insertion E249_A250insIMGHSHSGSCLL ${ }^{13}$ (henceforth designated as E249_A250insl-L) and a single-residue insertion A253_L254ins $N^{13}$ are located in ABD1 in the same $\mathrm{CH} 1-\mathrm{CH} 2$ connecting loop, which is disordered in both existing crystal structures of ABD cores of yeast and plant fimbrins. ${ }^{30}$ The remaining three missense mutations are located in the $\mathrm{CH} 2$ of $\mathrm{ABD} 1$ (A368D), ${ }^{12}$ in a proline-rich loop of $\mathrm{CH} 3(\mathrm{~N} 446 \mathrm{~S}){ }^{5}$ and another loop of $\mathrm{CH} 3$ in proximity to a tentative actin-binding surface ${ }^{31}$ (L478P). ${ }^{14}$

In this study, we characterized all five known insertion and missense Ol-linked PLS3 mutations leading to a full-length PLS3 protein production, as well as two frameshift mutations (T578Nfs X $4^{9}$ and $A 589 Q$ Qfs $X 21^{6}$ ), which, due to proximity to the $3^{\prime}$-untranslated region, may escape the nonsense-mediated mRNA decay $^{28,29}$ and result in truncated PLS3 variants (Fig. 1a). We provided molecular and cellular insights into the pathological effects of the PLS3 mutants. This analysis allowed classification of the mutations into four groups based on the resulting functional impairment: (1) PLS3 protein loss/instability (gene deletions or nonsense/frameshift mutations), (2) actin bundling-deficient
(L478P), (3) $\mathrm{Ca}^{2+}$-insensitive (E249_A250insl-L and A368D), and (4) $\mathrm{Ca}^{2+}$-hypersensitive (A253_L254insN and N446S). The data imply that the $\mathrm{Ca}^{2+}$-regulated F-actin bundling function of PLS3 is essential for bone formation. Despite the well-recognized role of $\mathrm{Ca}^{2+}$ in the regulation of vertebrate plastins in vitro, this is the first study, to our knowledge, showing that the fine-tuned $\mathrm{Ca}^{2+}$ regulation is crucial for cellular localization and functionality of these proteins. Importantly, location of the mutations perturbing $\mathrm{Ca}^{2+}$ sensitivity in both ABDs of the plastin core allowed us to map the position of the RD and propose a mechanistic explanation for the $\mathrm{Ca}^{2+}$-dependent inhibition of the plastin/fimbrin family of proteins.

\section{RESULTS}

Protein instability is a likely reason of OI phenotype in case of the frameshift but not the insertion and missense PLS3 mutations To check whether the OI phenotype can be a consequence of destabilized/denatured protein, seven recombinant OI PLS3 mutants and wild-type (WT) PLS3 (Fig. 1a, b) were expressed and purified from Escherichia coli. While the WT and five missense/ insertion PLS3 mutants were soluble, the two truncated variants carrying T578NfsX4 and A589QfsX21 frameshift mutations were largely insoluble (Supplementary Fig. S1a-c). Furthermore, 
Table 1. Biochemical properties of PLS3 variants carrying Ol-linked mutations

\begin{tabular}{|c|c|c|c|c|c|}
\hline PLS3 construct & $\mathrm{T}_{\mathrm{m}} /{ }^{\circ} \mathrm{C} \pm \mathrm{SE}$ & $\begin{array}{l}\mathrm{K}_{\mathrm{d}} / \mu \mathrm{mol} \cdot \mathrm{L}^{-1} \\
\pm \mathrm{SD}\end{array}$ & \multicolumn{2}{|c|}{$[\mathrm{PLS} 3]_{50 \%} / \mu \mathrm{mol} \cdot \mathrm{L}^{-1} \pm \mathrm{SD}$} & $\mathrm{pCa}_{50 \%} \pm \mathrm{SD}$ \\
\hline Wild type & $49.47 \pm 0.72$ & $7.41 \pm 0.10$ & $0.49 \pm 0.28$ & $2.97 \pm 1.48$ & $5.46 \pm 0.14$ \\
\hline N446S & $46.87 \pm 0.07$ & $4.79 \pm 0.56$ & $0.58 \pm 0.14$ & $6.36 \pm 2.46$ & $6.44 \pm 0.21$ \\
\hline A253_L254insN & $45.94 \pm 0.95$ & $3.77 \pm 0.19$ & $0.53 \pm 0.16$ & $2.09 \pm 1.55$ & $6.66 \pm 0.17$ \\
\hline
\end{tabular}

$T_{m}$ melting temperature determined by DSF, $K_{d}$ equilibrium dissociation constant of PLS3 binding to F-actin, $[P L S 3]_{50 \%}$ bundling efficiency of PLS3 (concentration of PLS3 at $50 \%$ of F-actin bundled), $\mathrm{pCa}_{50 \%} \mathrm{Ca}^{2+}$ sensitivity of PLS3 (- $\log \left[\mathrm{Ca}^{2+}\right]$ at $50 \%$ reduction in light scattering of actin bundles), SE standard error of the mean, $S D$ standard deviation of the mean

attempts to purify the truncation constructs from the minor soluble fraction failed due to their proteolytic degradation (Supplementary Fig. S1d). Thermal denaturation assessed by differential scanning fluorimetry (DSF) revealed that all seven mutations destabilized the protein to varying degrees, albeit the strongest destabilization was observed for the frameshift mutations (Supplementary Fig. S1e) further substantiating their disruptive nature. The five missense/insertion mutations resulted in moderate protein destabilization $\left(2.6^{\circ} \mathrm{C}-7.6^{\circ} \mathrm{C}\right.$, Fig. $1 \mathrm{C}$, Table 1), but the transition point did not drop below $42^{\circ} \mathrm{C}$ (i.e., was well above the physiological temperature), and no substantial increase in protein aggregation/degradation was observed. Accordingly, all five missense/insertion mutants expressed in human U2OS osteosarcoma cells were detected as a single full-length protein band on a western blot (Supplementary Fig. S7). Therefore, protein destabilization is a likely reason for the disease phenotype by the frameshift but not the missense/insertion mutations. Hence, we focused on characterization of two PLS3 insertion mutants (E249_A250insl-L and A253_L254insN) and three missense mutants (A368D, N446S, and L478P).

L478P mutation abolishes F-actin bundling by PLS3

F-actin binding and bundling abilities of PLS3 mutants were evaluated by differential co-sedimentation at high $(300000 \mathrm{~g})$ and low $(17000 \mathrm{~g})$ centrifugation speeds, respectively. ${ }^{27}$ All five mutants retained F-actin binding ability (Fig. 1d, Table 1), while the L478P mutation in ABD2 completely abolished the ability of PLS3 to bundle actin filaments (Fig. 1e), likely due to the proximity of the mutated residue to the F-actin binding site of $\mathrm{CH} 3$ (Fig. 1b). ${ }^{31}$

To gain structural insight into the effects of this bundlingdeficient mutation, we obtained a 3.9-Å resolution cryo-EM map of F-actin decorated by the ABD2 domain of plastin 2 (PLS2) using a single-particle method for reconstruction of helical filaments ${ }^{32}$ (Fig. 2a, Supplementary Fig. S2, Supplementary Table S1). The use of PLS2 ABD2 for the reconstruction was justified by (i) an inherent instability of PLS3 ABD2 rendering its purification unfeasible ${ }^{27}$ and (ii) a high overall level of sequence conservation in ABD2 of the two isoforms and $100 \%$ identity in the 474-483 loop (PLS3 numeration) encompassing the L478P mutation (Supplementary Fig. S3). In the obtained cryo-EM reconstruction, the core of filamentous actin has the highest resolution, but the PLS2 $\mathrm{CH} 3$ and $\mathrm{CH} 4$ domains are both well resolved in the map. The resolution is lower the further from the helical axis, especially in the $\mathrm{CH} 4$ domain, and is shown with the local resolution estimation (Supplementary Fig. S2). The $\mathrm{CH} 3$ domain is directly interacting with $\mathrm{F}$-actin, while $\mathrm{CH} 4$ domain has no contacts with F-actin. The superposition of F-actin in our reconstruction with a previously published pure F-actin structure (ADP state, PDB ID: 5ONV) shows that the RMSD of Ca atoms is only $0.8 \AA$ (Fig. 2b), which indicates there are no significant conformational changes in F-actin after the decoration by PLS2. In comparison with the crystal structures of the yeast and plant fimbrin cores, ${ }^{30}$ the $\mathrm{CH} 4$ domain in the PLS2 ABD2/F-actin complex is shifted outward to prevent it from clashing with F-actin (Fig. 2c). The loop 474-483 PLS3 $_{\text {(471-480 PLS2; }}$ hereafter, PLS2 numeration is provided in parentheses) encompassing the L478P mutation makes extensive contacts with the Dloop and the 81-96 helix of actin located in subdomains 2 and 1, respectively (Fig. $2 d$ ). The side chain of L478 (L475) does not form direct contacts with actin as it points away, towards the ABD2 core. Essentially, the side chain of L478 (L475) is involved in stabilization of the loop structure by forming a hydrophobic cluster with conserved V467 (V464), F476 (F473), 1481 (1478), and L497 (L494) residues (Fig. 2e, Supplementary Fig. S3, Supplementary Table S2). All these residues are conserved not only across ABD2 domains of different plastins/fimbrins, but also across ABDs of $\mathrm{t}-\mathrm{CH}$ proteins from filamin, a-actinin, spectrin, dystrophin, and utrophin families (Supplementary Fig. S3), where they contribute to the conformational stability of the respective loop (Fig. 2e). The L478P mutation is likely to distort this cluster, thus influencing the loop conformation and negatively affecting binding of $\mathrm{t}-\mathrm{CH}$ proteins to actin. On the other hand, of the nine residues of filamin A implicated in direct interaction with actin, ${ }^{33}$ only two are conserved in ABD2 of plastins (Fig. 2f, Supplementary Fig. S3), pointing to a considerable plasticity of the $\mathrm{t}-\mathrm{CH}$ interaction mode with F-actin.

Four Ol-linked PLS3 mutations impair $\mathrm{Ca}^{2+}$ sensitivity $\mathrm{Ca}^{2+}$ is recognized as a major signal transduction factor defining the sensitivity of osteocytes to mechanical stimuli in vitro and in vivo ${ }^{34-38}$ and is also involved in negative regulation of the actinbundling ability of plastins. $\mathrm{Ca}^{2+}$ ions bind to two EF-hands in the $\mathrm{RD}$ of the protein, whose location relative to the actin-binding core remains unknown. It is recognized, however, that upon binding to $\mathrm{Ca}^{2+}$, the EF-hands wrap around the adjacent $\mathrm{CBM}$, ${ }^{27,39}$ leading to inhibition of $A B D 2$ via an unknown mechanism.

Under saturating $\mathrm{Ca}^{2+}$ concentrations, the A253_L254insN responded to $\mathrm{Ca}^{2+}$ similarly to WT PLS3, while actin bundling by N446S was inhibited by $\mathrm{Ca}^{2+}$ about two times more effectively (Supplementary Fig. S4, Table 1). The N446S mutation is associated with a strong Ol phenotype in a heterozygous female patient $^{5}$ and, therefore, is likely dominant. Remarkably, both mutants showed about an order of magnitude increased sensitivity to $\mathrm{Ca}^{2+}\left(\mathrm{pCa}_{50 \%} 6.66 \pm 0.17\right.$ and $\left.6.44 \pm 0.21\right)$ compared with WT PLS3 $\left(\mathrm{pCa}_{50 \%} 5.46 \pm 0.14\right)$, as detected by a change in light scattering of actin-PLS3 bundles disassembled upon titration with $\mathrm{Ca}^{2+}$ (Fig. 1f, Table 1). The increased sensitivity of both mutants matches the $\mathrm{K}_{\mathrm{d}}$ of $\mathrm{Ca}^{2+}$ to the high-affinity PLS3 EF-hand $\left(\mathrm{K}_{\mathrm{d}} 0.37 \mu \mathrm{mol} \cdot \mathrm{L}^{-1}, 40\right.$ corresponding to $\left.\mathrm{pCa} 6.43\right)$, tentatively 
a

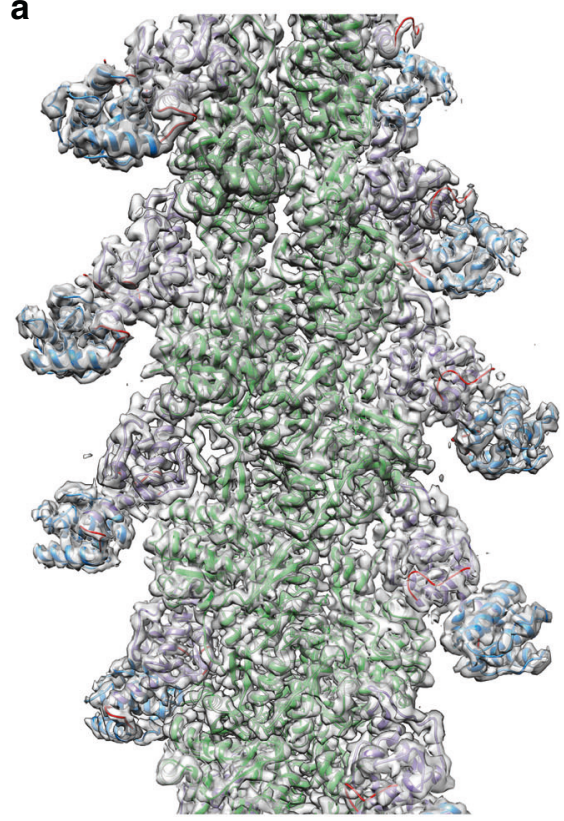

e

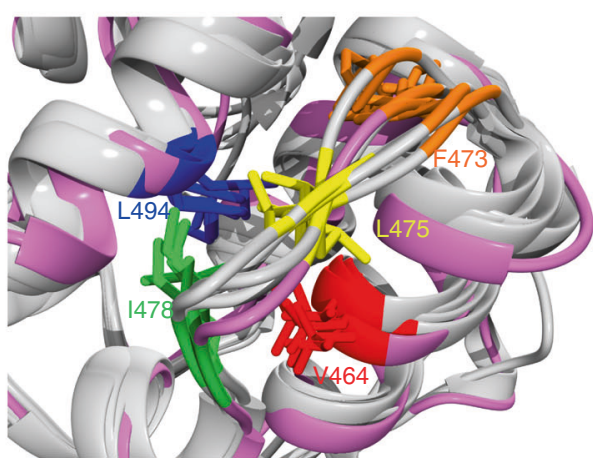

b

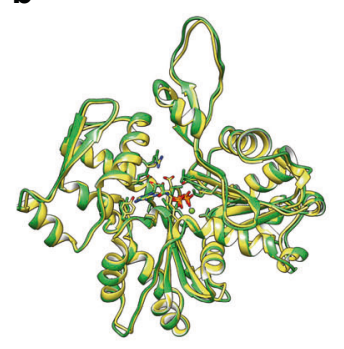

C
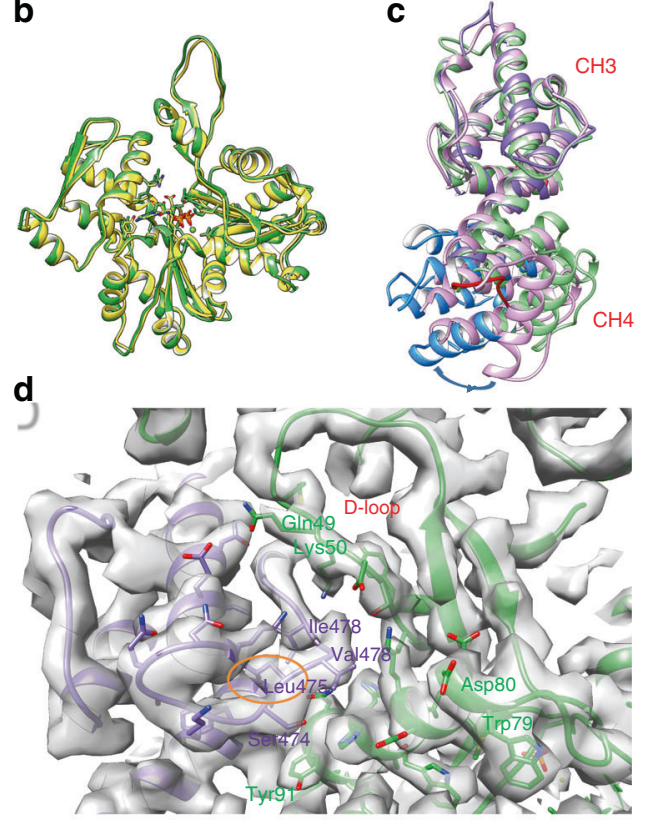

f

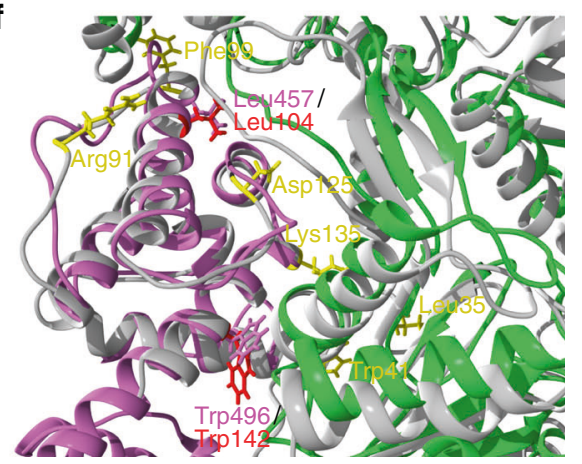

Fig. 2 Ol-linked L478P PLS3 mutation disrupts the actin-binding surface of ABD2. a Cryo-EM reconstruction with aligned model of ABD2 ${ }_{\text {PLS2- }}$ decorated F-actin (PDB ID: 6VEC; EMDB: EMD-21155). Actin is colored in green, $\mathrm{CH} 3$ and $\mathrm{CH} 4$ are in purple and blue, respectively. The loops linking the $\mathrm{CH}$ domains (385-394 and 511-517) are in red (see also Supplementary Fig. S2 and Supplementary Table S1). b Superposition of Factin decorated by ABD2 2 PLS2 (green; PDB ID: 6VEC) and pure F-actin (yellow; PDB ID: 5ONV). c Superposition of ABD2 2 PLS2 in the complex with F-actin (CH3 domain is in purple, $\mathrm{CH} 4$ domain is in blue; PDB ID: 6VEC) and crystal structures of fimbrin ABD2 from Arabidopsis thaliana (pink; PDB ID: 1PXY) and Schizosaccharomyces pombe (green; PDB ID: 1RT8). The arrow indicates the rotation of CH4 upon F-actin binding. $\mathbf{d}$ The interface of the PLS2 CH3 domain (purple; PDB ID: 6VEC) binding to F-actin, showing that L475 (corresponding to L478 in PLS3) is not directly interacting with F-actin (green). e Superposition of the L475-containing loop of WT-ABD2 $2_{\mathrm{PLS} 2}$ (in magenta) with the corresponding elements of $\mathrm{ABD}$ s from various $\mathrm{t}$-CH-domain proteins (in gray): utrophin, dystrophin, filamin $\mathrm{A}$, $\alpha$-actinin, and ABD2 of yeast fimbrin (PDB IDs are provided in the Supplementary Table S2). Distances between L475 and the other four members of the cluster (V464, F473, 1478, and L494) are consistent with hydrophobic interactions (see Supplementary Table S2). For respective multiple sequence alignment ${ }^{87}$ see Supplementary Fig. S3. f Superposition of F-actin-bound structure of $A B D 2_{P L S 2}$ obtained in the present study (purple; PDB ID: 6VEC) with filamin A structure (gray; PDB ID: 6D8C) produced by aligning actin (gray/green for $A B D 2_{P L S 2} /$ filamin A). Actin-binding residues in the $A B D$ of filamin $A^{33}$ that are not conserved with PLS2 ABD2 are shown in yellow. The only two actin-binding residues of filamin conserved in PLS2 ABD2 are in red; the respective conserved PLS2 residues are in magenta

suggesting that the mutations diminish the contribution of the low-affinity

EF-hand ( $\mathrm{K}_{\mathrm{d}} 10.6 \mu \mathrm{mol} \cdot \mathrm{L}^{-1},{ }^{40}$ corresponding to $\left.\mathrm{pCa} 4.97\right)$ to plastin inhibition.

In contrast, the E249_A250insl-L and A368D mutants virtually lost sensitivity to $\mathrm{Ca}^{2+}$, enabling bundling even in the presence of high $\mathrm{Ca}^{2+}$ concentrations (Fig. 1f, Supplementary Fig. S4, Table 1). Also, regardless of the presence of $\mathrm{Ca}^{2+}$, the bundling ability of the E249_A250insl-L mutant was enhanced compared with that of WT PLS3 (Fig. 1e, Supplementary Fig. S4, Table 1). Given that all four mutations are located away from the EF-hands, perturbed interaction of $\mathrm{RD}$ with the $\mathrm{ABD} 1-\mathrm{ABD} 2$ core is a likely reason of the impaired $\mathrm{Ca}^{2+}$ sensitivity.
Ol-linked PLS3 mutants show differential subcellular distribution Although PLS3 is ubiquitously expressed, all known developmental defects linked to PLS3 mutations are restricted to connective tissues: bones ${ }^{5-17}$ and fascia. ${ }^{26}$ Therefore, we focused on osteoblast (U2OS), osteocyte (Ocy454), and fibroblast (XTC) cell lines for PLS3 localization studies. In agreement with previous reports, ${ }^{20,21,41,42}$ mEmerald- or TagRFPT-tagged WT PLS3 was colocalized with the F-actin-rich elements at the cell edge, focal adhesions (FAs), and stress fibers (SFs) (Fig. 3a, b) of U2OS osteoblasts and Ocy454 osteocytes, while Ol mutants displayed differential subcellular distribution, grouped in accordance with their biochemical properties. Specifically, the L478P bundlingdeficient mutant showed diffuse localization, suggesting that 

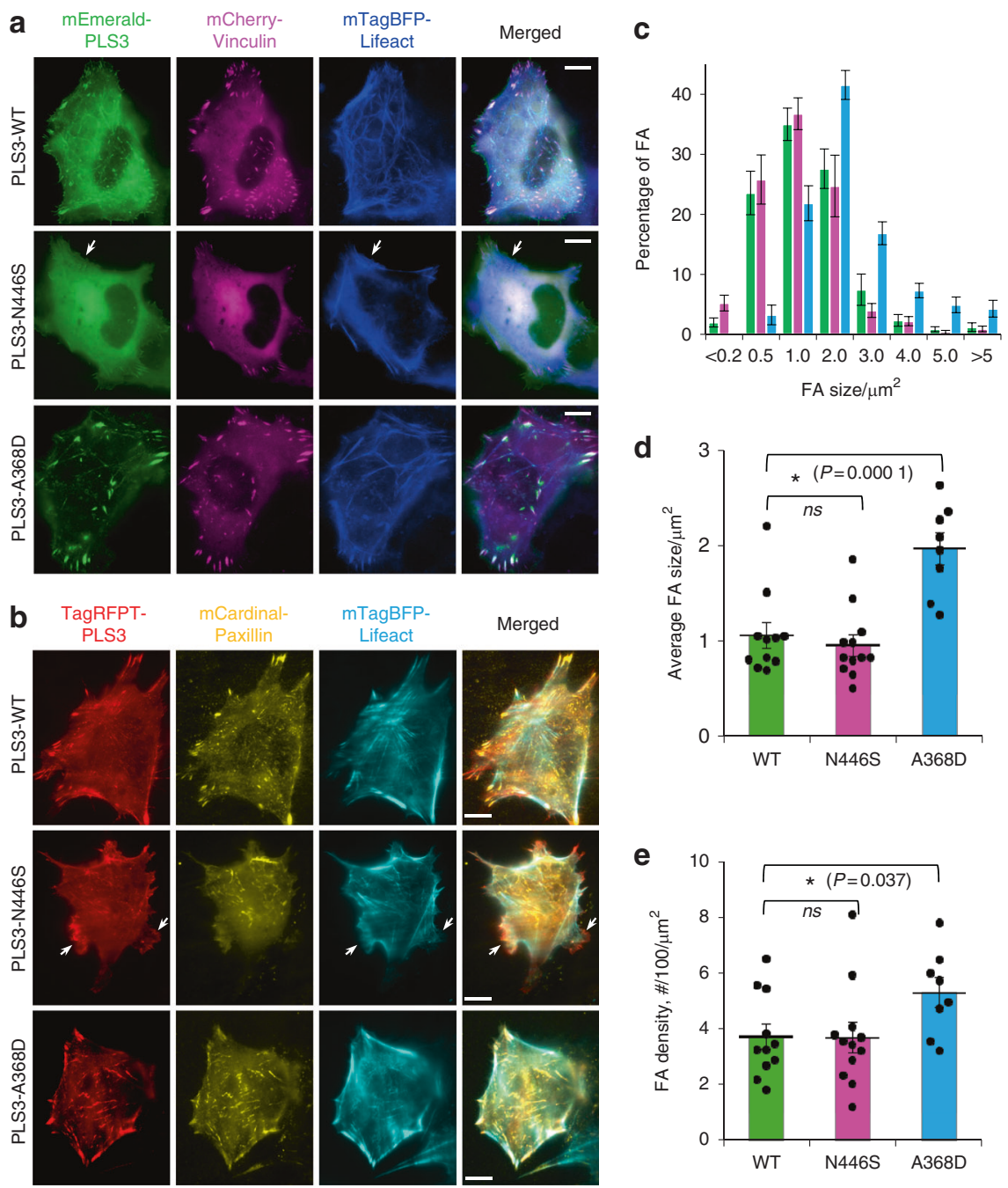

Fig. 3 Effects of Ol-linked PLS3 mutations on cellular localization of PLS3 and morphology of focal adhesions. a Live-cell total internal reflection fluorescence microscopy (TIRFM) imaging of U2OS osteoblasts co-transfected with mEmerald-PLS3 constructs (green), mCherryvinculin (FA, magenta) and mTagBFP-Lifeact (F-actin, blue). See also Supplementary Fig. S5a. b Live-cell TIRFM imaging of Ocy454 osteocytes co-transfected with TagRFPT-PLS3 constructs (red), mCardinal-paxillin (FA, yellow), and mTagBFP-Lifeact (F-actin, cyan). See also Supplementary Fig. S5b. White arrows in (a, b) point at membrane ruffles; scale bars are $10 \mu \mathrm{m}$. FA size (area) distribution (c), average FA sizes (area; (d)), and FA density (number of FA per cell area; (e)) were quantified in U2OS cells transiently transfected with mEmerald-PLS3 constructs and immunostained with anti-vinculin (for FA) and phalloidin (for F-actin). The color legend in $\mathbf{c}$ is the same as in $\mathbf{d}$ and $\mathbf{e}$. See also Supplementary Figs. S6 and S7. Number of analyzed cells is 11 for WT, 12 for N446S, and 8 for A368D (with 37-142 individual FAs per cell). Data are shown as means \pm SE, individual data points are plotted as circles. Statistical significance was determined by two-sample (equal variances) Student's $t$ test with two-tailed distribution, $n s$ is non-significant $(P>0.05)$

binding via $A B D 1$ alone is not sufficient to support its strong association with F-actin in any of the observed compartments (Supplementary Fig. S5). Both $\mathrm{Ca}^{2+}$-hypersensitive mutants showed reduced localization to FAs and SFs, but remained associated with the cell edge (Fig. 3a, b, Supplementary Fig. S5). Cells expressing these mutants displayed more prominent membrane ruffles. In striking contrast, both $\mathrm{Ca}^{2+}$-insensitive mutants vividly co-localized with FAs and SFs, whose morphology was both enhanced and distorted (Fig. 3a, b, Supplementary Fig. S5). Thus, the density and size of the FAs were significantly increased in cells expressing both $\mathrm{Ca}^{2+}$-insensitive mutants, while $\mathrm{Ca}^{2+}$-hypersensitive Ol mutants of PLS3 did not affect these parameters (Fig. 3c-e, Supplementary Fig. S6). The expression levels of PLS3 constructs were comparable in all cases (Supplementary Fig. S7) and could not account for the observed differences.

The observed phenotypes of PLS3 mutants were reproduced in spreading Xenopus laevis XTC fibroblasts, whose flat morphology, prominent lamellipodia, and high resistance to photodamage 43 make them particularly valuable for high-resolution live-cell microscopy. In XTC fibroblasts, mEmerald-tagged WT PLS3 was enriched at the leading edge, but also found moderately associated with FAs and SFs (Fig. 4a). Similarly to U2OS and Ocy454 cells, the bundling-deficient L478P mutant showed diffuse localization (Fig. 4b), whereas the $\mathrm{Ca}^{2+}$-hypersensitive mutants were highly enriched at the leading edge and almost excluded from the FAs and SFs (Fig. 4c, d). The $\mathrm{Ca}^{2+}$-insensitive mutants were depleted at the leading edge but strikingly enriched at FAs (Fig. 4e, f), which were notably larger than those in cells expressing WT or $\mathrm{Ca}^{2+}$-hypersensitive PLS3 mutants. Localization of PLS3 at the FA sites was partially overlapping with FA marker paxillin but extended toward the cell center; such a pattern was most reminiscent of the distribution of another $\mathrm{t}-\mathrm{CH}$-domain actin bundler a-actinin (Fig. 4g).

Considering the differential subcellular distribution of $\mathrm{Ca}^{2+}$-hyper- and hyposensitive PLS3 OI mutants, $\mathrm{Ca}^{2+}$ appeared 

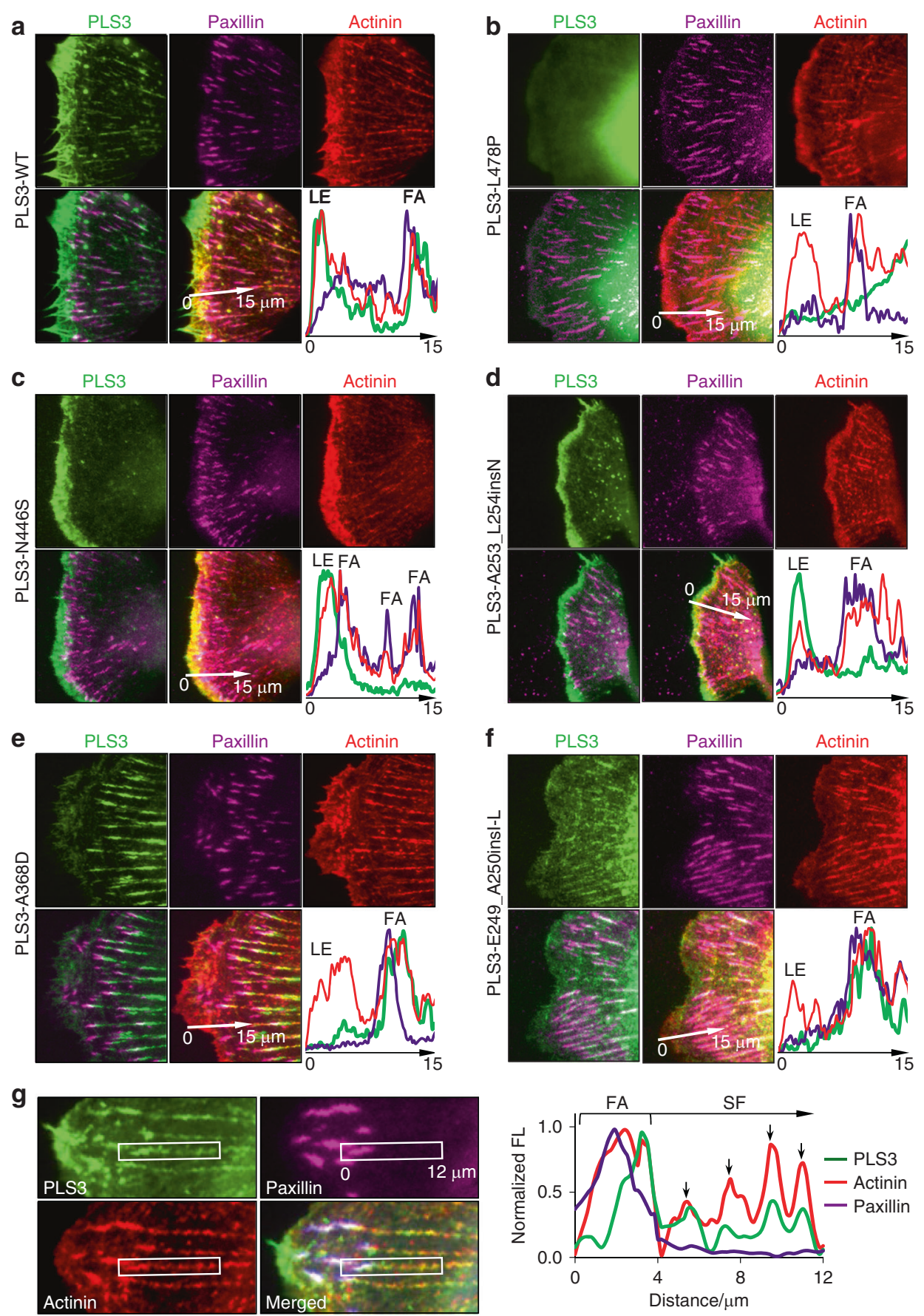

Fig. 4 Differential distribution of OI-linked PLS3 mutants in spreading XTC fibroblasts. a-f Localization of PLS3 variants at the cell periphery was assessed by TIRFM in live XTC cells co-transfected with mEmerald-PLS3 constructs, mCardinal-paxillin, and mCherry-actinin. For each PLS3 construct, sets of individual channel images along with two-channel (PLS3 and paxillin) and three-channel (PLS3, paxillin, and actinin) overlays are shown. Graphs represent fluorescence intensity profiles along the $15-\mu \mathrm{m}$-long lines indicated by white arrows; plot colors match those of the corresponding fluorescent channels. Note differential distribution of OI PLS3 mutants between the leading edge (LE) and FAs. g Colocalization of mEmerald-PLS3-WT with FAs (mCardinal-paxillin) and SFs (mCherry-actinin) in cells with high levels of expression cultured in $\mathrm{Ca}^{2+}$-free medium. In the boxed areas, identical horizontal $12-\mu \mathrm{m}$-long lines were drawn (centrally, along the long axis) in each channel starting from the FA through the actin SF. Line intensity profiles show co-localization of PLS3 with actinin patches on the SFs and at the FAs (note that PLS3 localization is shifted towards the cell center)

to be a key factor regulating recycling of PLS3 from actin bundles at the FA sites to the branched network of actin filaments at the leading edge of spreading/migrating cells. To verify this hypothesis, we examined the dynamics of WT PLS3 and representative PLS3 OI mutants upon depletion of intracellular $\mathrm{Ca}^{2+}$ caused by the addition of EGTA to the medium. Notable redistribution of PLS3 from the leading edge to FAs was observed for both WT and N446S $\mathrm{Ca}^{2+}$-hypersensitive mutant, with the only difference that the leading-edge depletion was more prominent for WT PLS3, while FA enrichment was more notable for the N446S mutant 
a

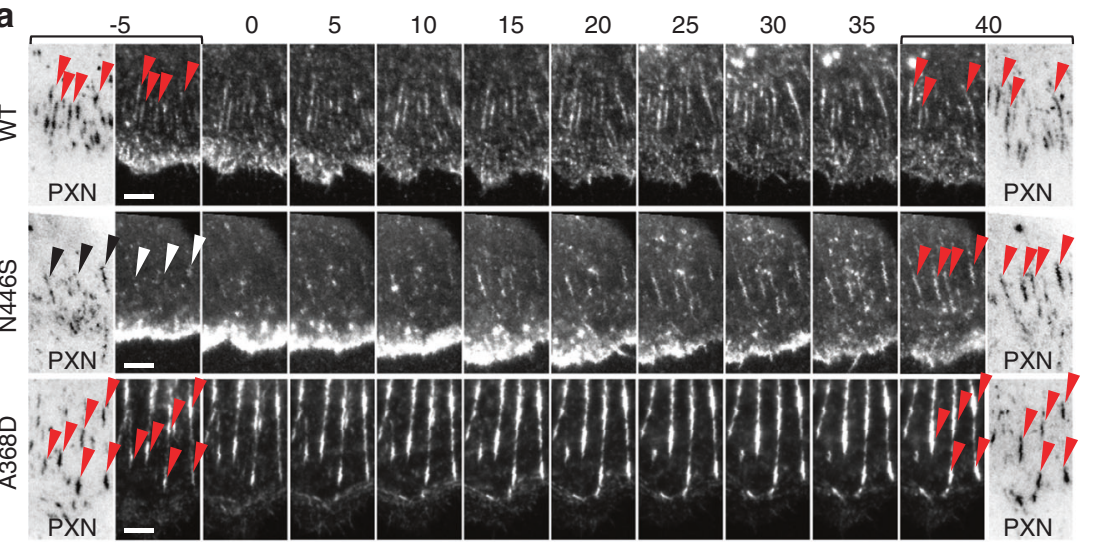

b

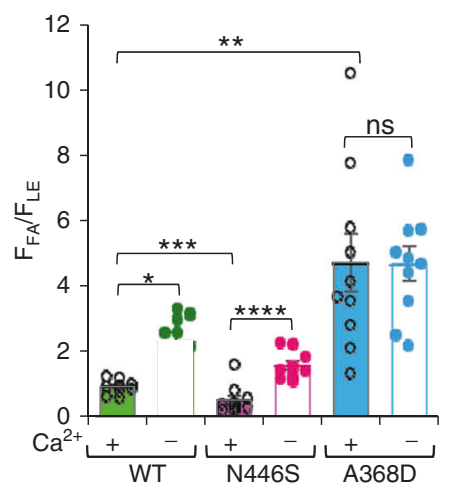

C

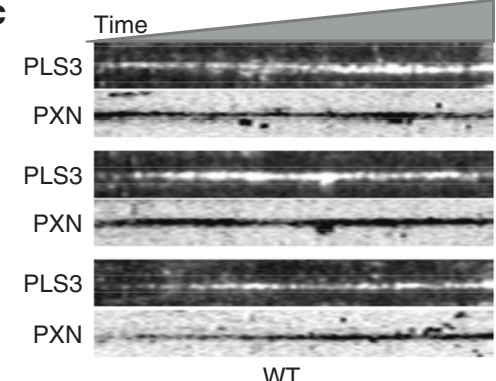

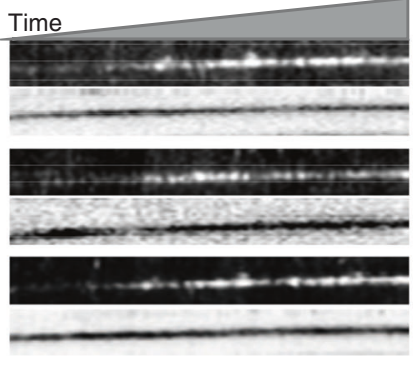

N446S

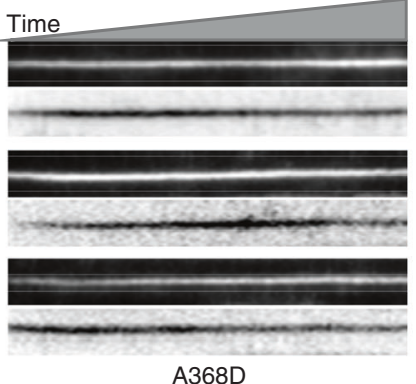

A368D

Fig. 5 Effects of $\mathrm{Ca}^{2+}$ depletion on PLS3 redistribution to focal adhesion sites. a Time-lapse TIRFM images of XTC cells co-transfected with representative $\mathrm{Ca}^{2+}$-hypo- and hypersensitive mEmerald-PLS3 constructs and mCardinal-paxillin were recorded upon chelation of $\mathrm{Ca}^{2+}$ in the culture medium with $5 \mathrm{mmol} \cdot \mathrm{L}^{-1}$ EGTA. Time before and after the addition of EGTA is indicated in minutes. mCardinal-paxillin is shown on inverted images (black signal on white background) marked PXN. Red arrowheads point to PLS3-positive FAs; black and white arrowheads denote PLS3-negative FAs. Scale bar is $5 \mu \mathrm{m}$ (see also Supplementary Movie S1). b Ratios of PLS3 fluorescence at focal adhesions ( $\left.\mathrm{F}_{\mathrm{FA}}\right)$ to that of at the leading edge $\left(\mathrm{F}_{\mathrm{LE}}\right)$ in the $\mathrm{Ca}^{2+}$-containing medium (final extracellular concentration of $\mathrm{Ca}^{2+} 3.5 \mathrm{mmol} \cdot \mathrm{L}^{-1}$ ) and upon $\mathrm{Ca}^{2+}$ chelation by $5 \mathrm{mmol} \cdot \mathrm{L}^{-1}$ EGTA. Data are shown as means $\pm S E$, individual data points are plotted as circles. Number of analyzed cells is $n=10$ for each condition. Statistical significance was determined by two-sample (equal variances) Student's $t$ test with two-tailed distribution: ${ }^{*} P=1.4 \times 10^{-2}$, ${ }^{* *} P=4.5 \times 10^{-4}$, ${ }^{* * *} p=$ $8.9 \times 10^{-5}, * * * * P=2.6 \times 10^{-5}$, ns is non-significant $(p>0.05)$. c Representative kymographs (length-time scans) display' PLS3 and paxillin (PXN) fluorescence intensities over time along the lines drawn through individual FAs upon $\mathrm{Ca}^{2+}$ chelation with $5 \mathrm{mmol}^{-L^{-1}}$ EGTA

(Fig. 5, Supplementary Movie S1). A moderate enrichment at the FA sites was visually detectable for the hyposensitive A368D mutant (Fig. 5a, b), but was found to be statistically insignificant (Fig. 5c).

Mapping the position of the RD on the surface of PLS3 core To date, high-resolution structures of full-length plastin/fimbrin do not exist, and, therefore, the location of RD relative to the core is unknown and the mechanism of plastin inhibition by $\mathrm{Ca}^{2+}$ is not understood. Remarkably, all four PLS3 Ol mutations with impaired $\mathrm{Ca}^{2+}$ sensitivities are grouped near the same face of the ABD core (Fig. 1b), while two of them are even located in the same $\mathrm{CH} 1-\mathrm{CH} 2$ loop (E249_A250insI-L and A253_L254insN). We speculated that the perturbed $\mathrm{Ca}^{2+}$ sensitivity of the four mutants suggests that the mutations are at or near the RD/core interface (Figs. $1 \mathrm{~b}$ and $6 \mathrm{a}$, dashed oval). To test this hypothesis, we used full-length and RDtruncated $(\triangle \mathrm{RD})$ PLS3 constructs to evaluate solvent accessibility of single-cysteine residues incorporated into the core loops facing the tentative RD-binding region (Fig. 6a, dashed oval) and involved in osteoporosis (i.e., 240-268 and 446-456 loops harboring E249_A250insl-L, A253_L254insN, and N446S Ol mutations). Singlecysteine PLS3 mutants K452C and A250C in constructs containing $\mathrm{RD}$ incorporated a Cys-selective acrylodan probe approximately two times slower than their counterparts without RD (Fig. 6b, c), consistent with a reduced solvent accessibility of the loops due to their proximity to $\mathrm{RD}$. $\mathrm{Ca}^{2+}$ did not affect the modification rates of A250C and only mildly influenced the modification of K452C, suggesting that the $\mathrm{Ca}^{2+}$-induced conformational rearrangements only moderately affect the RD position.
In addition to the osteoporosis loop A250C and K452C mutants, we also tested the $\mathrm{I388C}$ and T391C constructs with lone cysteines located in a long linker loop between ABD1 and ABD2 (i.e., 377-394 loop; Fig. 6a). Labeling of both I388C and T391C with acrylodan was very slow regardless of the presence of RD (Fig. $6 \mathrm{~d}$, e) suggesting their overall buried position.

\section{DISCUSSION}

F-actin bundling is the primary physiological function of plastins The majority of the Ol-linked PLS3 mutations are either nonsense or frameshift mutations resulting in premature termination codons. Such mutations rarely result in translated protein products due to nonsense-mediated mRNA decay, a quality control measure that degrades mRNA containing premature stopcodons preventing the generation of truncated proteins. ${ }^{28,29}$ While mRNAs can avoid nonsense-mediated decay, ${ }^{44}$ none of the canonical mechanisms apply to the majority of the identified OI mutations. Accordingly, the two studies that evaluated the in vivo expression of truncated OI PLS3 mutants, did not detect protein products. $^{13,16}$ Furthermore, were the nonsense and frameshift mutants to be translated, they would not be fully functional as only three would retain protein sequence through $\mathrm{CH} 3$ domain, while $\mathrm{CH} 4$ domain also contributes to F-actin bundling. ${ }^{27}$ Our analysis confirmed substantial destabilization and aggregation/ denaturation for the longest truncated OI PLS3 variants, which could be produced due to the frameshift mutations T578NfsX4 and $\mathrm{A} 589 \mathrm{Qfs} \mathrm{X} 21$ introducing premature termination codons 49 and 20 amino acids before the PLS3 natural stop codon. 
a

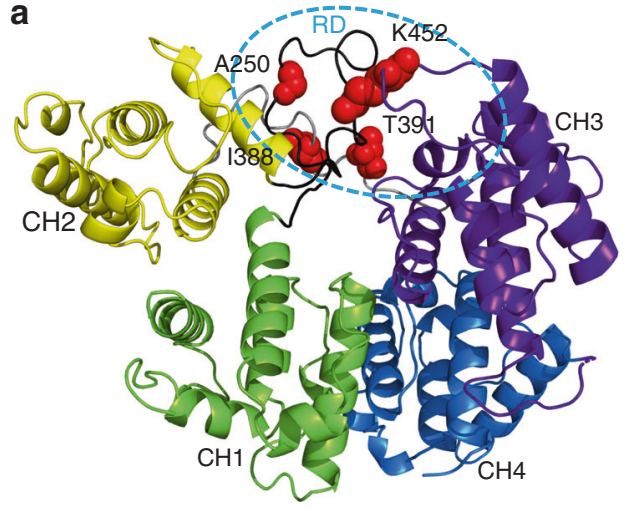

b

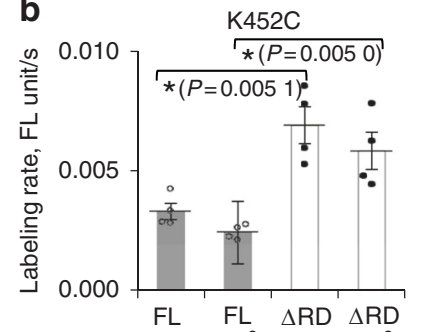

EGTA $\stackrel{\mathrm{Ca}}{\mathrm{C}} \stackrel{\Delta \mathrm{RD}}{\mathrm{EGTA}} \stackrel{\mathrm{CR}}{\mathrm{Ca}^{2+}}$

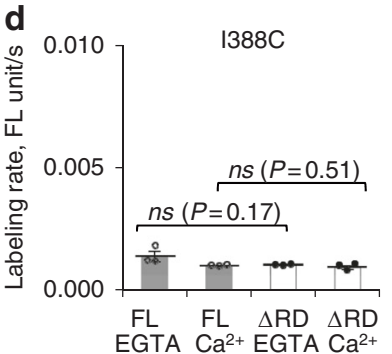

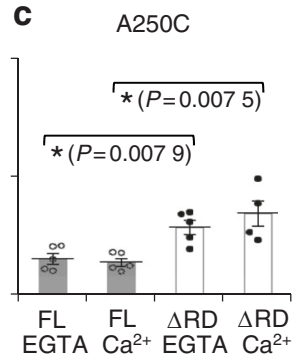

e

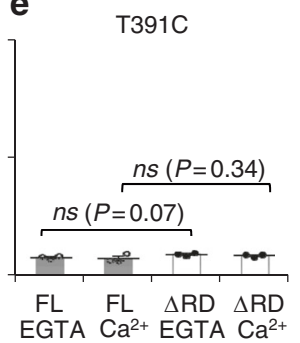

Fig. 6 Mapping the position of the regulatory domain on the surface of PLS3 core. a Positions of single-cysteine mutations in the loops harboring Ol-associated mutations (A250C in the 240-268 and K452C in the 446-456 loops) and in the ABD1-ABD2-connecting linker (I388C and T391C in the 377-394 linker) are shown in red on a homology-based model of PLS3 (Phyre $2^{86}$; color scheme as in Fig. 1 ). The proposed position of RD is encircled by a dashed line. b-e Acrylodan labeling of the cysteine residues described in (a) introduced as the only cysteines into the full-length PLS3 (FL) and PLS3 lacking the $\mathrm{Ca}^{2+}$-binding regulatory domain $(\triangle \mathrm{RD})$. Labeling was assessed for each construct in the presence of either $1 \mathrm{mmol} \cdot \mathrm{L}^{-1}$ EGTA or $\mathrm{CaCl}_{2}$. The rates were calculated by fitting the data to a pseudo-first order kinetic model and plotted as means \pm SE ( $n=4$ for $\mathbf{b}, n=5$ for $\mathbf{c}, n=3$ for $\mathbf{d}$, e); individual data points are plotted as circles. Statistical significance was determined by twosample (equal variances) Student's $t$ test with two-tailed distribution

Therefore, in this study we focused on the five missense and insertion OI PLS3 mutations that are expected to produce fulllength protein products and, according to our DSF data, do not result in substantial protein destabilization. Of these five mutants, L478P lacks the bundling ability and, therefore, is functionally most similar to the Ol-linked PLS3 deletion and truncation mutants and most clearly connected to the disease phenotype. Plastins can bind to F-actin via their ABD1 domain independent of bundling ${ }^{27}$ and ABD1 alone is sufficient to positively affect Arp2/3mediated actin dynamics in vitro. ${ }^{45}$ Possible explanations for this observation are a competitive replacement of tropomyosin enabling a higher filament recycling rate by cofilin, ${ }^{46}$ or, on the contrary, stabilization of filaments via competition with cofilin. ${ }^{45}$ Yet, despite the unperturbed binding of L478P PLS3 via ABD1 to actin, the mutation still leads to osteoporosis and appears to be insufficient for the association of the mutant with either FAs or the lamellipodia (Figs. 3 and 4). Plastins are well recognized to stabilize parallel aligned F-actin bundles. ${ }^{47}$ Of the two major PLS3-enriched cellular structures, FAs contain parallel actin bundles, while the leading-edge actin cytoskeleton is enriched in branched actin networks depleted in parallel bundles. It remained unclear, until now, whether plastins are associated with the leading-edge actin cytoskeleton in an F-actin binding mode (i.e., through ABD1) or in a bundling mode (i.e., through both $A B D$ domains). These data correlate with the essential role of fimbrin's (a yeast plastin orthologue) actin-bundling activity in endocytosis at the actin patches, ${ }^{46,48}$ which originate as the Arp2/3 complex-organized branched actin networks. Our findings suggest that binding via both ABD1 and ABD2 is essential for PLS3 localization both at the leading edge and FAs and corroborate that F-actin bundling is the primary physiological function of plastins at both locations.

OI mutations unbalance PLS3 regulation by $\mathrm{Ca}^{2+}$

Despite the well-recognized role of $\mathrm{Ca}^{2+}$ in plastin regulation in vitro, how the EF-hands domain is positioned on the core is unclear, and the mechanism of the inhibition is not understood. We found that two of the five characterized Ol-causative PLS3 mutants lost $\mathrm{Ca}^{2+}$ sensitivity and remained constitutively active even in the presence of $\mathrm{Ca}^{2+}$, while two others became more sensitive to $\mathrm{Ca}^{2+}$. While the former is a typical 'loss of function' effect, as the RD of plastin loses its ability to inhibit bundling in the presence of $\mathrm{Ca}^{2+}$, the latter 'gain of function' effect (a higher sensitivity to $\mathrm{Ca}^{2+}$ ) is much less common and calls for at least a speculative explanation. In contrast to PLS2, whose both EF-hand domains share the same $\sim 1-\mu \mathrm{mol} \cdot \mathrm{L}^{-1} \mathrm{~K}_{\mathrm{d}}$ to $\mathrm{Ca}^{2+}$, PLS3 EF-hands vary in their affinity to $\mathrm{Ca}^{2+}$ by nearly thirty-fold. ${ }^{40}$ The measured sensitivity levels of the hypersensitive mutants $\left(\mathrm{pCa}_{50 \%}=6.5-6.7\right)$ match the $0.37-\mu \mathrm{mol} \cdot \mathrm{L}^{-1} \mathrm{~K}_{\mathrm{d}}$ of the high-affinity binding PLS3 EFhand ( pCa 6.4). This observation tentatively suggests that while $\mathrm{Ca}^{2+}$ binding to both EF-hands is required for inhibition of WT PLS3, single high-affinity site binding is sufficient to inhibit the hypersensitive mutants. It is tempting to speculate that a similar effect, achieved via a posttranslational modification or caused by a binding partner, may represent a physiological mechanism of tuning PLS3 $\mathrm{Ca}^{2+}$ sensitivity. Interestingly, the abnormally high level of PLS3 $\mathrm{Ca}^{2+}$ sensitivity leading to osteoporosis is nearly identical to the physiological sensitivities of PLS2 and PLS1, ${ }^{27}$ indicating that the $\mathrm{Ca}^{2+}$ regulation of the isoforms is tuned to their tissue-specific functions.

Ol mutations point on the location of the RD

The 240-268 and 446-456 loops harboring three of the mutants with perturbed $\mathrm{Ca}^{2+}$ sensitivity, although belonging to different ABDs, are located on the same surface within $\sim 20 \AA$ of each other (Fig. 1b), a distance that can be easily bridged by RD $(\sim 35 \AA)$. The proposed arrangement is consistent with covalent crosslinking of RD to both ABD1 and ABD2. ${ }^{27}$ Similarly, the presence of RD limits solution accessibility of the loops as deduced from less effective acrylodan labeling of cysteine residues embedded into the loops (Fig. 6b, c), further supporting proximity of the osteoporosis loops to the RD. The fourth mutation (A368D), although not in these loops, faces both the 224-239 helix (preceding the 240-268 loop) and the 123-137 helix (following the linker between RD and $A B D 1)$ and, therefore, may unbalance $\mathrm{Ca}^{2+}$ regulation by affecting 

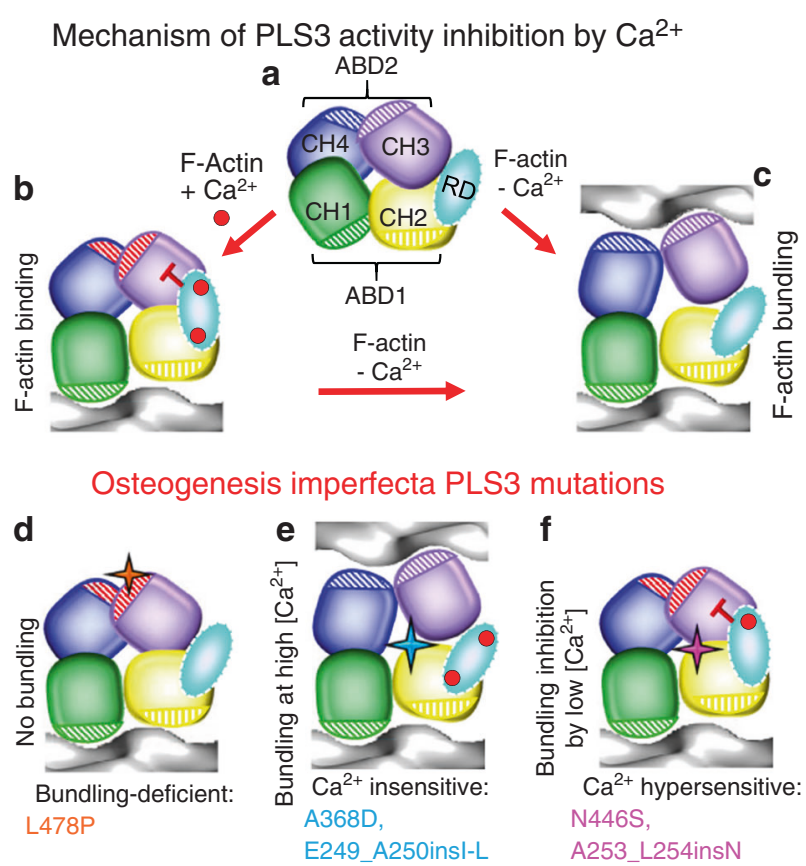

Fig. 7 Model of plastin regulation by $\mathrm{Ca}^{2+}$. a Schematic representation of plastin domains (the color scheme and domain designation as in Fig. 1). $\mathbf{b}$ In the presence of $\mathrm{Ca}^{2+}$, plastins bind to actin via $A B D 1$ only, while $C H 3-C H 4$ of $A B D 2$ are locked in the inhibited state (red dashed) by stapling ABD2 to ABD1 by RD. c Dissociation of $\mathrm{Ca}^{2+}$ releases the RD-applied constraints to ABD2 allowing its binding to actin and resulting in F-actin bundling. The OI PLS3 mutations lead to osteoporosis by either directly disrupting the actin-binding site of ABD2 (L478P; (d)), inhibiting the ABD2 by sub-physiological $\mathrm{Ca}^{2+}$ concentrations (N446S and A253_L254insN; (f)), or by disabling the inhibition by physiological $\mathrm{Ca}^{2+}$ concentrations (A368D and E249_A250insl-L; (e)), preventing normal functions of PLS3

both RD and an RD-binding site on the 240-268 loop. The unstructured nature of the $240-268$ loop $^{30}$ also indirectly supports its involvement in binding to the RD. Indeed, disordered protein regions are notoriously engaged in polymorphic interactions ${ }^{49}$ and may effectively mediate binding to several partners ${ }^{50}$ or to different conformational states of the same partner such as the $\mathrm{Ca}^{2+}$-free and -bound states of RD.

Mechanistic model of the $\mathrm{Ca}^{2+}$-dependent regulation of PLS3 activity

Proximity of the linkers connecting the $\mathrm{CH}$ domains to the proposed RD-binding site (Fig. 6a) suggests that RD may affect Factin binding by restraining the relative orientation of the $\mathrm{CH}$ domains within $\mathrm{ABD} 2(\mathrm{CH} 3$ to $\mathrm{CH} 4)$, between $\mathrm{ABDs}(\mathrm{CH} 2$ to $\mathrm{CH} 3)$, or both. Based on the results of the current and recent studies, we propose the following model of plastin regulation by $\mathrm{Ca}^{2+}$ (Fig. 7). In the presence of $\mathrm{Ca}^{2+}$, plastins bind to actin via ABD1 only, while $\mathrm{CH} 3-\mathrm{CH} 4$ are locked in the inhibited state by RD, which restricts the rearrangements required for the $\mathrm{CH} 3$ binding to actin (Fig. 2c) by fastening ABD2 to ABD1. Dissociation of $\mathrm{Ca}^{2+}$ leads to separation of the EF-hands from the CBM, unwinding of the CBM helix to a relaxed structure, ${ }^{39}$ and release of the ABD1-ABD2 constraint. In line with this model, the OI PLS3 mutations lead to osteoporosis by either (i) directly disrupting the actin-binding site of ABD2 (L478P), (ii) inhibiting the interaction by low, noninhibitory for WT PLS3, $\mathrm{Ca}^{2+}$ concentrations (N446S, A253_L254insN), or (iii) by disabling the inhibition by physiological $\mathrm{Ca}^{2+}$ concentrations (A368D, E249_A250insl-L). Such dysregulation leads to either weakening or, on the contrary, nonphysiological enforcement of FA sites by respectively accelerating or preventing the PLS3 recycling to the cell periphery.

Links between PLS3 function, calcium signaling, and osteoporosis Although the exact role of PLS3 in osteogenesis is currently unclear, numerous indirect lines of evidence suggest its contribution to sensing and transduction of mechanical stimuli. Of the three cell types immediately involved in constructing and remodeling the bone, the role of PLS3 in bone resorption by osteoclasts is rather convoluted, given that the major plastin isoform in these cells is PLS2 and the presence of PLS3 was not clearly demonstrated. Nevertheless, a recent work has proposed a link between PLS3, NFKB signaling, and the regulation of osteoclast activity. ${ }^{51}$ Another study has found no effect of PLS3 mutations on the osteoclast activity. ${ }^{14}$

In contrast, PLS3 is the predominant isoform in the other two major bone cell types, osteoblasts and osteocytes. Moreover, its expression in osteoblasts is increased upon maturation and PLS3 is highly enriched at the bifurcations of osteocyte dendritic processes, ${ }^{52}$ which are recognized as mechanotransducing elements. ${ }^{52,53}$ The ability of osteoblasts and especially osteocytes to sense and respond to mechanical stimuli is the key factor defining bone morphogenesis and remodeling directly (by affecting the synthetic activity of osteoblasts) and indirectly (by controlling the activity and apoptosis of both osteoblasts and osteoclasts by humoral factors). ${ }^{54-56}$

In osteoblasts and osteocytes, rise of cytoplasmic $\mathrm{Ca}^{2+}$ is linked to bone development via calcineurin/NFAT ${ }^{57}$ and noncanonical $\mathrm{Wnt}^{53}$ pathways, the latter of which is recognized as a central signaling pathway in osteogenesis. Furthermore, the rise of cytoplasmic $\mathrm{Ca}^{2+}$ in these cells can be caused by mechanical stimuli, ${ }^{37,58}$ which is an essential component of mechanically controlled bone formation and remodeling by both osteoblasts and osteocytes. Although the mechanisms of osteoblast/osteocyte mechanosensitivity are debated, integrin/adhesion-dependent activation of voltage-gated calcium channels are thought to be involved. ${ }^{37,58-60}$ Association of plastins with integrin-based FAs and its sensitivity to $\mathrm{Ca}^{2+}$ furthers the link between PLS3 and the mechanotransduction machinery. Local $\mathrm{Ca}^{2+}$ spikes associated with FAs are recognized in other cell types (e.g., fibroblasts ${ }^{61}$ and astrocytes ${ }^{62}$ ) and may represent a common mechanism of cell response to mechanical stimuli. In astrocytes, this results in accelerated recycling of FA sites via recruitment of focal adhesion kinase (FAK). ${ }^{63}$ The importance of FAK and FA dynamics in bone development is illustrated by a potent suppression of mechanically induced bone regeneration in FAK-deficient mice. ${ }^{64}$ Our finding that $\mathrm{Ca}^{2+}$ promotes dissociation of PLS3 from FAs and SFs, allowing its recycling to the leading edge, may represent $a$ parallel, FAK-independent mechanism of FA dynamics regulation.

Overall, the reduced or increased sensitivity to $\mathrm{Ca}^{2+}$ of four out of five Ol-associated PLS3 mutations characterized in the present study as well as their respectively perturbed localization in osteoblast, osteocyte, and fibroblast cells indicate that the delicately controlled $\mathrm{Ca}^{2+}$-dependent regulation of actin bundling by PLS3 is essential for normal bone formation.

\section{MATERIALS AND METHODS}

Plasmid construction

Recombinant plastins were cloned as $\mathrm{N}$-terminally 6xHis-tagged constructs into a modified pColdl vector (Clontech) containing a TEV protease recognition site ${ }^{27}$ using NEBuilder HiFi DNA Assembly Master Mix (New England Biolabs). Site-directed mutagenesis was carried out based on Quick-change Site-directed Mutagenesis strategy (Agilent Technologies) using Q5 DNA polymerase and Dpnl restriction enzyme (New England Biolabs). QuikChange Lightning Multi Site-Directed Mutagenesis Kit (Agilent Technologies) was used for multisite mutagenesis. 
Protein expression and purification

Actin was purified from chicken skeletal muscle acetone powder obtained from flash-frozen chicken breasts (Trader Joe's) ${ }^{65}$ as previously described. ${ }^{66}$ Actin was stored up to 1 month on ice in G-buffer $\left(5 \mathrm{mmol} \cdot \mathrm{L}^{-1}\right.$ Tris- $\mathrm{HCl} \quad[\mathrm{pH} \quad 8.0], 0.2 \mathrm{mmol} \cdot \mathrm{L}^{-1} \quad \mathrm{CaCl}_{2}$, $0.2 \mathrm{mmol} \cdot \mathrm{L}^{-1}$ ATP, $5 \mathrm{mmol} \cdot \mathrm{L}^{-1} \beta$-mercaptoethanol, $0.1 \mathrm{mmol} \cdot \mathrm{L}^{-1}$ phenylmethanesulfonyl fluoride [PMSF], and $0.01 \% \mathrm{NaN}_{3}$ ) with dialysis against fresh G-buffer after 2 weeks.

Plastins were expressed in BL21-CodonPlus(DE3) E. coli (Agilent Technologies) grown in nutrient-rich media (1.25\% tryptone, $2.5 \%$ yeast extract, $125 \mathrm{mmol} \cdot \mathrm{L}^{-1} \mathrm{NaCl}, 0.4 \%$ glycerol, and $50 \mathrm{mmol} \cdot \mathrm{L}^{-1}$ Tris- $\mathrm{HCl}[\mathrm{pH} 8.2])$. Upon reaching the $\mathrm{OD}_{600}$ of $1-1.2$, the cells were cooled to $15^{\circ} \mathrm{C}$ and expression was induced by the addition of $1 \mathrm{mmol} \cdot \mathrm{L}^{-1}$ isopropyl $\beta$-D-1-thiogalactopyranoside. Cells were maintained at $15^{\circ} \mathrm{C}$ for $16 \mathrm{~h}-24 \mathrm{~h}$ before harvest by centrifugation. Plastin mutants were purified by immobilized metal affinity chromatography on TALON cobalt resin (Clontech) under native conditions. Elution was carried out by the addition of $250 \mathrm{mmol} \cdot \mathrm{L}^{-1}$ imidazole, $50 \mathrm{mmol} \cdot \mathrm{L}^{-1}$ 4-(2-hydroxyethyl)-1-piperazineethanesulfonic acid (HEPES; $\mathrm{pH} 7.4$ ), $300 \mathrm{mmol} \cdot \mathrm{L}^{-1} \mathrm{NaCl}$, and $0.1 \mathrm{mmol} \cdot \mathrm{L}^{-1}$ PMSF. Purified plastins were dialyzed overnight against PLS buffer ( $10 \mathrm{mmol} \cdot \mathrm{L}^{-1} \mathrm{HEPES}[\mathrm{pH} 7.0], 30 \mathrm{mmol} \cdot \mathrm{L}^{-1} \mathrm{KCl}$, $2 \mathrm{mmol} \cdot \mathrm{L}^{-1} \mathrm{MgCl}_{2}, 0.5 \mathrm{mmol} \cdot \mathrm{L}^{-1}$ ethylene glycol-bis( $\beta$-aminoethyl

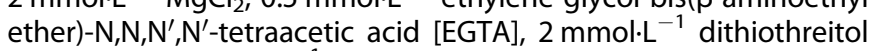
[DTT], and $0.1 \mathrm{mmol} \cdot \mathrm{L}^{-1} \mathrm{PMSF}$ ) before being flash-frozen in liquid nitrogen and stored at $-80^{\circ} \mathrm{C}$.

Protein labeling with acrylodan

Single-cysteine mutations were introduced on the Cys-null background in the full-length and $\triangle$ RD PLS3 constructs. Proteins were reduced in the presence of $10 \mathrm{mmol} \cdot \mathrm{L}^{-1} \mathrm{DTT}$ for $1 \mathrm{~h}$ on ice followed by DTT removal by passing through a NAP5 desalting column (GE Healthcare) equilibrated with buffer containing $10 \mathrm{mmol} \cdot \mathrm{L}^{-1} \mathrm{HEPES}\left(\mathrm{pH} 7.0\right.$ ) and $30 \mu \mathrm{mol} \cdot \mathrm{L}^{-1} \mathrm{KCl}$. Labeling with acrylodan was carried out as previously described. ${ }^{67}$ Briefly, reduced PLS3 constructs were labeled under pseudo-first-order conditions with a 1:100 acrylodan to PLS3 molar ratio $\left(0.05 \mathrm{mmol} \cdot \mathrm{L}^{-1}\right.$ acrylodan and $\left.5 \mu \mathrm{mol} \cdot \mathrm{L}^{-1} \mathrm{PLS} 3\right)$ in the buffer above with the addition of $1 \mathrm{mmol} \cdot \mathrm{L}^{-1}$ either EGTA or $\mathrm{CaCl}_{2}$. Labeling rate was monitored at $25^{\circ} \mathrm{C}$ using a PTI QuantaMaster 400 spectrofluorometer (Horiba) with excitation and emission wavelengths of 385 and $465 \mathrm{~nm}$, respectively.

Differential scanning fluorimetry (DSF)

DSF was conducted as reported previously. ${ }^{68,69}$ Briefly, plastins were diluted to $3 \mu \mathrm{mol} \cdot \mathrm{L}^{-1}$ in PLS buffer in the presence of 1:5 000 dilution of Sypro Orange dye (Invitrogen). Changes in fluorescence of the dye, which preferentially binds to protein hydrophobic regions exposed upon temperature-induced unfolding, were measured in triplicates using a CFX Real-Time PCR Detection System (Bio-Rad). The melting temperatures $\left(T_{m}\right)$ were determined as the maximum of the first derivative of each normalized experimental curve and expressed as the mean of three independent repetitions \pm standard error (SE).

F-actin binding and bundling co-sedimentation assays $\mathrm{G}$-actin was switched from a $\mathrm{Ca}^{2+}$ - to $\mathrm{Mg}^{2+}$-bound form by the addition of $0.1 \mathrm{mmol} \cdot \mathrm{L}^{-1} \mathrm{MgCl}_{2}$ and $0.5 \mathrm{mmol} \cdot \mathrm{L}^{-1} \mathrm{EGTA}$ and incubated on ice for $10 \mathrm{~min}$. Subsequently, actin polymerization was induced by the addition of $\mathrm{MgCl}_{2}, \mathrm{KCl}$, and HEPES (pH 7.0) to 2,30 , and $10 \mathrm{mmol} \cdot \mathrm{L}^{-1}$, respectively. Polymerization was carried out for $30 \mathrm{~min}$ at room temperature before addition of plastin. In actin-binding assays, plastins were added to a final concentration of $5 \mu \mathrm{mol} \cdot \mathrm{L}^{-1}$, and actin was varied from 1 to $50 \mu \mathrm{mol} \cdot \mathrm{L}^{-1}$. For actin-bundling assays, actin was used at $5 \mu \mathrm{mol} \cdot \mathrm{L}^{-1}$ while the plastin concentrations were varied from 0.1 to $15 \mu \mathrm{mol} \cdot \mathrm{L}^{-1}$. Where indicated, $\mathrm{CaCl}_{2}$ was added to yield a concentration of free $\mathrm{Ca}^{2+}$ of $0.5 \mathrm{mmol} \cdot \mathrm{L}^{-1}$ determined as described. ${ }^{70}$ Reactions were incubated overnight at $4{ }^{\circ} \mathrm{C}$ followed by $1 \mathrm{~h}$ at $25^{\circ} \mathrm{C}$ prior to centrifugation. Binding reactions were centrifuged at $300000 \mathrm{~g}$ and $25^{\circ} \mathrm{C}$ for $30 \mathrm{~min}$. Bundling reactions were centrifuged at 17 $000 \mathrm{~g}$ and $25^{\circ} \mathrm{C}$ for $15 \mathrm{~min}$. Supernatants were separated from pellets and analyzed by SDS-PAGE stained with Coomassie Brilliant Blue. Densitometry analysis was performed using ImageJ software. $^{71,72}$

$\mathrm{K}_{\mathrm{d}} \mathrm{s}$ were determined using the binding isotherm equation:

Fraction PLS Bound $=\frac{P+A+K_{d}-\sqrt{\left(P+A+K_{d}\right)^{2}-4 P A}}{2 P}$,

where $P$ is the concentration of plastin and $A$ is the concentration of F-actin.

Bundling efficiency was calculated by fitting to the Hill equation:

Percentage of Actin Bundled $=\frac{[P L S]^{n}}{K_{A}^{n}+[P L S]^{n}}$,

where $n$ is the Hill coefficient and $K_{A}{ }^{n}$ is the concentration of plastin at $50 \%$ actin bundled.

Binding and bundling experiments were conducted in at least three and two independent repetitions, respectively, and the final values were presented as mean values with SE or standard deviations (SD) as indicated in the figure and table legends.

Light scattering assay

To determine $\mathrm{Ca}^{2+}$ sensitivity of PLS3 constructs, light scattering by PLS3-induced F-actin bundles was measured at $90^{\circ}$ to the incident light using a PTI QuantaMaster 400 spectrofluorometer (Horiba). Both excitation and emission wavelengths were set at $350 \mathrm{~nm}$ with $3 \mathrm{~nm}$ slits. Actin and plastin (5 and $1 \mu \mathrm{mol} \cdot \mathrm{L}^{-1}$, respectively) were mixed in PLS buffer and incubated overnight at $4{ }^{\circ} \mathrm{C}$ followed by incubation for $1 \mathrm{~h}$ at $25^{\circ} \mathrm{C}$ and degassing for 15 min under vacuum. $\mathrm{Ca}^{2+}$ was increased incrementally and measurements were taken for at least 1 min between the titration points and averaged for each increment. The resulting values were normalized and the average of each of three independent experiments $\pm S E$ was plotted versus free $\mathrm{pCa}$ in solution. The $\mathrm{pCa}_{50 \%}$ values were calculated by fitting the data to a logistic curve using Origin software (OriginLab).

\section{Cryo-EM data collection, helical reconstruction, and model} building

To prepare the F-actin/ABD2 ${ }_{\mathrm{PLS} 2}$ complex, skeletal muscle actin was polymerized as previously described ${ }^{31}$; ABD2 of PLS2 was added at a fourfold excess to $1-\mu \mathrm{mol} \cdot \mathrm{L}^{-1} \mathrm{~F}$-actin. A $1.5 \mu \mathrm{L}$ sample of the F-actin/ABD2 complex was applied to discharged lacey carbon grids, followed by plunge freezing with a Leica EM GP. Movies were collected in a Titan Krios cryo-electron microscope at $300 \mathrm{keV}$ equipped with a Falcon III direct electron detector, sampling at $1.4 \AA /$ pixel. The defocus range was set to $1.5-2.5 \mu \mathrm{m}$, with a total dose of $\sim 55$ electrons/ $/ \AA^{2}$. MotionCor 2 was used to motion correct and dose-weight all the movies, followed by CTF estimation of the aligned images using the CTFFIND3 program. ${ }^{73}$ Images with poor CTF estimation were eliminated. The e2helixboxer program in the EMAN2 ${ }^{74}$ software package was used for boxing the long filaments. Subsequently, the filaments were extracted and cut into shorter segments in Relion 3.0, ${ }^{75}$ which generated $248184256 \mathrm{px}$-long overlapping segments. A subgroup ( $49 \%$ of the full dataset) of nicely ABD2-decorated F-actin was selected after 2D and 3D classification. We further used AutoRefine followed by polishing, as well as CtfRefine in Relion 3.0. The final symmetry converged to twist of $-166.5^{\circ}$ and rise of $28.0 \AA$ with local symmetry searches. The final resolution was determined by both map:map FSC as well as model:map FSC. The map:map FSC yielded a resolution of $3.9 \AA$ at FSC $=0.143$, and the 
model:map FSC also showed a resolution of $3.9 \AA$ at $\mathrm{FSC}=0.38$ (which is $\sqrt{0.143) .}$

We used a cryo-EM structure of actin in complex with ADP (PDB ID: 5ONV) and a PLS2 homology model based on a template (PDB ID: 1RT8) from SWISS-MODEL ${ }^{76}$ as initial models. The subunit models of actin and ABD2 of PLS2 were first fit as rigid bodies into the $3.9 \AA$ cryo-EM map using UCSF Chimera, ${ }^{77}$ followed by model refinement using RosettaCM. ${ }^{78}$ The top ten models were selected and refined by COOT $^{79}$ and PHENIX. ${ }^{80}$ The best model was then symmetrically built by using Rosetta, followed by PHENIX real space refinement. The final model was validated by MolProbity ${ }^{81}$ (Table S1). The cryo-EM map was deposited with accession code EMD-21155 in the Electron Microscopy Data Bank (EMDB), and the coordinates were deposited in the Protein Data Bank (PDB) with accession code PDB ID: 6VEC. Molecular graphics were performed using PyMol Molecular Graphics System (version 2) and UCSF Chimera. ${ }^{77}$

Cell culture

U2OS human osteosarcoma cells (RRID:CVCL_0042) were maintained in DMEM supplemented with $10 \%$ fetal bovine serum (FBS; Corning), $100 \mu \mathrm{g} \cdot \mathrm{mL}^{-1}$ streptomycin, $100 \mu \mathrm{g} \cdot \mathrm{mL}^{-1}$ penicillin, $2 \mathrm{mmol} \cdot \mathrm{L}^{-1}$ glutamine at $37^{\circ} \mathrm{C}$ in a humidified atmosphere containing $5 \% \mathrm{CO}_{2}$. Ocy454 osteocytic cells (RRID:CVCL_UW31) ${ }^{82}$ derived from a double transgenic mouse expressing GFP under the control of dentin matrix protein 1 promoter and SV40 large T antigen, were obtained from Bone Cells Core services (Boston University) and grown on plates coated with rat-tail collagen I $\left(5 \mu \mathrm{g} \cdot \mathrm{cm}^{-2}\right.$; Corning) in a-MEM medium (Gibco) supplemented with $10 \% \mathrm{FBS}, 100 \mu \mathrm{g} \cdot \mathrm{mL}^{-1}$ streptomycin, $100 \mu \mathrm{g} \cdot \mathrm{mL}^{-1}$ penicillin at $33^{\circ} \mathrm{C}$ in a humidified atmosphere containing $5 \% \mathrm{CO}_{2}$. X. laevis XTC-2 fibroblasts (RRID:CVCL_5610) were cultured in 70\% L-15 medium containing $10 \% \mathrm{FBS}, 100 \mu \mathrm{g} \cdot \mathrm{mL}^{-1}$ streptomycin, $100 \mu \mathrm{g} \cdot \mathrm{mL}^{-1}$ penicillin at $25^{\circ} \mathrm{C}$ at ambient humidity and $\mathrm{CO}_{2}$. Human U2OS cell line was authenticated by microsatellite genotyping (Genomics Shared Resource, OSU Comprehensive Cancer Center) with $100 \%$ match using The Cellosaurus cell line database. ${ }^{83}$ All cell lines were mycoplasma-free as tested by PCRbased mycoplasma detection analysis. ${ }^{84}$

Transient transfection

Plastin constructs fused at their C-terminus with either mEmerald or TagRFPT were cloned into a pcDNA3.1 vector (Invitrogen) using NEBuilder HiFi DNA Assembly Master Mix (New England Biolabs). mCherry-Alpha-Actinin-19, mCherry-Vinculin-23, mTagBFP-Lifeact7 , and mCardinal-Paxillin-22 ${ }^{85}$ were gifts from Michael Davidson (Addgene 54975, 55159, 54596, and 56171, respectively). Transient transfections were carried out using Turbofect transfection reagent (Thermo Fisher Scientific) according to manufacturer instructions.

\section{Immunofluorescence}

U2OS cells transiently transfected with mEmerald-PLS3 constructs were plated on 96 -well ibiTreat $\mu$-plate (ibidi 89626). After $24 \mathrm{~h}$ of transfection cells were fixed with $4 \%$ paraformaldehyde in phosphate buffered saline (PBS; Sigma) for $10 \mathrm{~min}$, permeabilized with $0.1 \%$ of Triton X-100 in PBS for $5 \mathrm{~min}$, blocked in $1 \%$ bovine serum albumin in PBS for 30 min, stained with rabbit anti-vinculin (Bethyl A302-535A-T; 1:200) followed by TRITC-conjugated antirabbit (Sigma T6778, 1:500), contra-stained with coumarinphalloidin (Santa Cruz Biotechnology sc-301532; $0.2 \mu \mathrm{mol} \cdot \mathrm{L}^{-1}$ final) and imaged using wide-field epifluorescence and Nikon CFI Plan Apochromat $\lambda \times 60$ oil objective (NA 1.40) on a Nikon Eclipse Ti-E microscope. Vinculin-positive FAs were counted in PLS3-mEmerald-transfected cells (number of analyzed cells was 11 for WT PLS3, 12 for N446S PLS3, and 8 for A368D PLS3, with 37-142 individual FAs per cell) and sizes of individual FA sites were measured using Image J2 $^{72}$
Western blotting

U2OS cells transfected with mEmerald-PLS3 constructs were harvested and cell lysates were prepared in the reducing SDSPAGE sample buffer. Samples were subjected to PAGE and transferred to nitrocellulose. Membranes were blocked in PBS containing $0.1 \%$ Tween-20 and $5 \%$ nonfat dry milk for $1 \mathrm{~h}$ at room temperature and incubated with anti-PLS3 antibody (Sigma SAB2700266; $1: 1000$ ) in the blocking buffer overnight at $4{ }^{\circ} \mathrm{C}$. Following three washes with PBS containing 0.1\% Tween-20, membranes were incubated with anti-rabbit antibody conjugated with horseradish peroxidase (HRP; Sigma A0545; 1:10 000) for $1 \mathrm{~h}$ at room temperature. Signal was detected using chemiluminescent HRP substrate WesternBright Sirius (Advansta) in an Omega Lum G imager (Aplegen).

Live-cell imaging

XTC cells in six-well plates at $70 \%$ confluence were co-transfected with the mEmerald-PLS3 constructs, mCherry-actinin, and mCardinal-paxillin. Prior to live-cell imaging, transfected cells were trypsinized and plated on poly-D-lysine (PDL) coated coverslips (Neuvitro GG-25-1.5-PDL) in Attofluor chambers (Invitrogen) in 70\% L-15 media lacking phenol red and FBS. Cells were allowed to spread for $30 \mathrm{~min}$ at $25^{\circ} \mathrm{C}$ and imaged at ambient conditions using Nikon TIRF module and a CFI Plan Apochromat $\lambda$ $\times 100$ oil objective (NA 1.45) on a Nikon Eclipse Ti-E microscope equipped with perfect focus system. Laser power was set to $15 \%$ and the field diaphragm was partially closed to minimize the cell area exposure and photodamage during the image acquisition. For $\mathrm{Ca}^{2+}$ removal experiments, $5 \mathrm{mmol} \cdot \mathrm{L}^{-1}$ EGTA was added to the culture medium to chelate $\mathrm{Ca}^{2+}$ and images were recorded with 30-s intervals. Image processing and assessment of the ratio of PLS3-mEmerald fluorescence signal at the cell edge and in FAs were performed using ImageJ. ${ }^{72}$ Kymographs and Supplementary Movie S1 were generated using Multiple Kymographs and Multi Stack Montage plugins of ImageJ, respectively.

U2OS and Ocy454 cells were plated on collagen I-coated PDL coverslips in phenol red-free serum-containing L-15 or a-MEM medium, respectively. U2OS cells were co-transfected with the mEmerald-PLS3 constructs, mCherry-vinculin, and mTagBFP-Lifeact, while GFP-positive Ocy454 cells were co-transfected with the TagRFPT-PLS3 constructs, mCardinal-paxillin, and mTagBFPLifeact. After $24 \mathrm{~h}$ of transfection, live-cell TIRFM imaging was conducted as described above.

\section{Statistical analysis}

Each experiment was conducted in 2-5 independent repetitions and final values were presented as mean values with SE or SD as indicated in the corresponding figure/table legends alone with the exact number of the conducted experiments/quantified events $(n)$. Two-sample (equal variance) Student's $t$ test with two-tailed distribution was used for data comparison ( $P$ values $<0.05$ were considered statistically significant). Data were graphed using KaleidaGraph (Synergy Software) and Excel (Microsoft Office); Origin software (OriginLab) was used for data fitting.

\section{ACKNOWLEDGEMENTS}

This work was supported by the American Cancer Society Institutional Research Grant (to D.S.K.), NIH GM114666 (to D.S.K.) and GM122510 (to E.H.E.), and the Ohio State University Cancer Comprehensive Center Pelotonia Graduate Fellowship (to C.L.S.). Any opinions, findings, and conclusions expressed in this material are those of the authors and do not necessarily reflect those of $\mathrm{NIH}$ and the Pelotonia Fellowship Program.

\section{AUTHOR CONTRIBUTIONS}

C.L.S., E.K., W.Z., E.H.E. and D.S.K. designed the study. C.L.S., E.K., W.Z., M.O. and L.A.R. conducted the experiments. C.L.S., E.K., W.Z., M.O., L.A.R., H.S., E.H.E. and D.S.K., 
analyzed the data. D.S.K. and E.H.E. supervised the project. C.L.S., E.K., D.S.K., W.Z. and E.H.E. wrote the paper. All authors commented on the paper.

\section{ADDITIONAL INFORMATION}

The online version of this article (https://doi.org/10.1038/s41413-020-0095-2) contains supplementary material, which is available to authorized users.

Competing interests: The authors declare no competing interests.

\section{REFERENCES}

1. Ensrud, K. E. \& Crandall, C. J. Osteoporosis. Ann. Intern Med 167, ITC17-ITC32 (2017).

2. Van Dijk, F. S. \& Sillence, D. O. Osteogenesis imperfecta: clinical diagnosis, nomenclature and severity assessment. Am. J. Med Genet A 164A, 1470-1481 (2014).

3. Lindahl, K. et al. Genetic epidemiology, prevalence, and genotype-phenotype correlations in the Swedish population with osteogenesis imperfecta. Eur. J. Hum. Genet 23, 1042-1050 (2015).

4. Forlino, A., Cabral, W. A., Barnes, A. M. \& Marini, J. C. New perspectives on osteogenesis imperfecta. Nat. Rev. Endocrinol. 7, 540-557 (2011).

5. Kampe, A. J. et al. PLS3 sequencing in childhood-onset primary osteoporosis identifies two novel disease-causing variants. Osteoporos. Int. 28, 3023-3032 (2017).

6. Balasubramanian, M. et al. Novel PLS3 variants in X-linked osteoporosis: exploring bone material properties. Am. J. Med. Genet. A 176, 1578-1586 (2018).

7. Costantini, A. et al. A novel frameshift deletion in PLS3 causing severe primary osteoporosis. J. Hum. Genet. 63, 923-926 (2018).

8. Chen, T. et al. Clinical, genetics, and bioinformatic characterization of mutations affecting an essential region of PLS3 in patients with BMND18. Int J. Endocrinol. 2018, 8953217 (2018).

9. Kannu, P., Mahjoub, A., Babul-Hirji, R., Carter, M. T. \& Harrington, J. PLS3 mutations in X-Linked osteoporosis: clinical and bone characteristics of two novel mutations. Horm. Res Paediatr. 88, 298-304 (2017).

10. Lv, F. et al. A novel large fragment deletion in PLS3 causes rare X-linked earlyonset osteoporosis and response to zoledronic acid. Osteoporos. Int. 28, 2691-2700 (2017).

11. Laine, C. M. et al. A novel splice mutation in PLS3 causes X-linked early onset lowturnover osteoporosis. J. Bone Miner. Res. 30, 437-445 (2015).

12. Nishi, E. et al. Exome sequencing-based identification of mutations in nonsyndromic genes among individuals with apparently syndromic features. Am. J. Med. Genet. Part A 170, 2889-2894 (2016).

13. van Dijk, F. S. et al. PLS3 mutations in X-linked osteoporosis with fractures. N. Engl. J. Med. 369, 1529-1536 (2013).

14. Fahiminiya, S. et al. Osteoporosis caused by mutations in PLS3: clinical and bone tissue characteristics. J. Bone Miner. Res. 29, 1805-1814 (2014).

15. Kampe, A. J. et al. PLS3 deletions lead to severe spinal osteoporosis and disturbed bone matrix mineralization. J. Bone Miner. Res. 32, 2394-2404 (2017).

16. Wang, L. et al. A novel nonsense variant in PLS3 causes X-linked osteoporosis in a Chinese family. Ann. Hum. Genet. 84, 92-96 (2020).

17. Cao, Y. J., Zhang, H. \& Zhang, Z. L. Novel mutations in the Wnt1, Tmem38b, P4hb, and Pls3 genes in four unrelated Chinese families with osteogenesis imperfecta. Endocr. Pract. 25, 230-241 (2019).

18. Shinomiya, H. Plastin family of actin-bundling proteins: its functions in leukocytes, neurons, intestines, and cancer. Int. J. cell Biol. 2012, 213492 (2012).

19. Lin, C. S., Park, T., Chen, Z. P. \& Leavitt, J. Human plastin genes: comparative gene structure, chromosome location, and differential expression in normal and neoplastic cells. J. Biol. Chem. 268, 2781-2792 (1993).

20. Brun, C. et al. T-plastin expression downstream to the calcineurin/NFAT pathway is involved in keratinocyte migration. PloS one 9, e104700 (2014).

21. Hagiwara, M. et al. Interaction of activated Rab5 with actin-bundling proteins, Land T-plastin and its relevance to endocytic functions in mammalian cells. Biochem. Biophys. Res. Commun. 407, 615-619 (2011).

22. Ikeda, $\mathrm{H}$. et al. The role of T-fimbrin in the response to DNA damage: silencing of T-fimbrin by small interfering RNA sensitizes human liver cancer cells to DNAdamaging agents. Int. J. Oncol. 27, 933-940 (2005).

23. Wottawa, M. et al. Hypoxia-stimulated membrane trafficking requires T-Plastin. Acta Physiol. 221, 59-73 (2017).

24. Yorgan, T. A. et al. Mice lacking plastin-3 display a specific defect of cortical bone acquisition. Bone 130, 115062 (2020).

25. Dor-On, E. et al. T-plastin is essential for basement membrane assembly and epidermal morphogenesis. Sci. Signal. 10, pii: eaal3154 (2017).
26. Boycott, K. M. \& Innes, A. M. 39th Annual David W. Smith workshop on malformations and morphogenesis: abstracts of the 2018 annual meeting. Am J Med Genet A, 674-746 (2019).

27. Schwebach, C. L., Agrawal, R., Lindert, S., Kudryashova, E. \& Kudryashov, D. S. The roles of actin-binding domains 1 and 2 in the calcium-dependent regulation of actin filament bundling by human plastins. J. Mol. Biol. 429, 2490-2508 (2017).

28. Kishor, A., Fritz, S. E. \& Hogg, J. R. Nonsense-mediated mRNA decay: the challenge of telling right from wrong in a complex transcriptome. Wiley Interdiscip. Rev. RNA 10, e1548 (2019).

29. Miller, J. N. \& Pearce, D. A. Nonsense-mediated decay in genetic disease: friend or foe? Mutat. Res. Rev. Mutat. Res. 762, 52-64 (2014).

30. Klein, M. G. et al. Structure of the actin crosslinking core of fimbrin. Structure 12, 999-1013 (2004).

31. Galkin, V. E., Orlova, A., Cherepanova, O., Lebart, M. C. \& Egelman, E. H. Highresolution cryo-EM structure of the F-actin-fimbrin/plastin ABD2 complex. Proc. Natl Acad. Sci. USA 105, 1494-1498 (2008).

32. Egelman, E. H. A robust algorithm for the reconstruction of helical filaments using single-particle methods. Ultramicroscopy 85, 225-234 (2000).

33. Iwamoto, D. V. et al. Structural basis of the filamin A actin-binding domain interaction with F-actin. Nat. Struct. Mol. Biol. 25, 918-927 (2018).

34. Rosenberg, N., Rosenberg, O. \& Soudry, M. Osteoblasts in bone physiology-mini review. Rambam Maimonides Med. J. 3, e0013 (2012).

35. Lewis, K. J. et al. Osteocyte calcium signals encode strain magnitude and loading frequency in vivo. Proc. Natl Acad. Sci. USA 114, 11775-11780 (2017).

36. Genetos, D. C., Geist, D. J., Liu, D., Donahue, H. J. \& Duncan, R. L. Fluid shearinduced ATP secretion mediates prostaglandin release in MC3T3-E1 osteoblasts. J. Bone Miner. Res. 20, 41-49 (2005).

37. $\mathrm{Cao}, \mathrm{C}$. et al. Increased $\mathrm{Ca}^{2+}$ signaling through $\mathrm{CaV} 1.2$ promotes bone formation and prevents estrogen deficiency-induced bone loss. JCl insight 2, pii: 95512 (2017).

38. Lu, X. L., Huo, B., Park, M. \& Guo, X. E. Calcium response in osteocytic networks under steady and oscillatory fluid flow. Bone 51, 466-473 (2012).

39. Ishida, H., Jensen, K. V., Woodman, A. G., Hyndman, M. E. \& Vogel, H. J. The calcium-dependent switch Helix of L-Plastin regulates actin bundling. Sci. Rep. 7, 40662 (2017).

40. Miyakawa, T. et al. Different $\mathrm{Ca}(2)(+)$-sensitivities between the EF-hands of T- and L-plastins. Biochem. Biophys. Res. Commun. 429, 137-141 (2012).

41. Li, N. et al. Actin-bundling protein plastin 3 is a regulator of ectoplasmic specialization dynamics during spermatogenesis in the rat testis. FASEB J. 29, 3788-3805 (2015)

42. Lyon, A. N. et al. Calcium binding is essential for plastin 3 function in Smndeficient motoneurons. Hum. Mol. Genet. 23, 1990-2004 (2014).

43. Watanabe, N. Fluorescence single-molecule imaging of actin turnover and regulatory mechanisms. Methods Enzymol. 505, 219-232 (2012).

44. Dyle, M. C., Kolakada, D., Cortazar, M. A. \& Jagannathan, S. How to get away with nonsense: mechanisms and consequences of escape from nonsense-mediated RNA decay. Wiley Interdiscip. Rev. RNA 11, e1560 (2020).

45. Giganti, A. et al. Actin-filament cross-linking protein T-plastin increases Arp2/3mediated actin-based movement. J. Cell Sci. 118, 1255-1265 (2004).

46. Skau, C. T. et al. Actin filament bundling by fimbrin is important for endocytosis, cytokinesis, and polarization in fission yeast. J. Biol. Chem. 286, 26964-26977 (2011).

47. Glenney, Jr,J. R., Kaulfus, P., Matsudaira, P. \& Weber, K. F-actin binding and bundling properties of fimbrin, a major cytoskeletal protein of microvillus core filaments. J. Biol. Chem. 256, 9283-9288 (1981).

48. Christensen, J. R. et al. Competition between topomyosin, fimbrin, and ADF/ Cofilin drives their sorting to distinct actin filament networks. eLife 6, pii: e23152 (2017).

49. Fong, J. H. et al. Intrinsic disorder in protein interactions: insights from a comprehensive structural analysis. PLoS Comput Biol. 5, e1000316 (2009).

50. Uversky, V. N. Intrinsic disorder-based protein interactions and their modulators. Curr. Pharm. Des. 19, 4191-4213 (2013).

51. Neugebauer, J. et al. Plastin 3 influences bone homeostasis through regulation of osteoclast activity. Hum. Mol. Genet. 27, 4249-4262 (2018).

52. Kamioka, H., Sugawara, Y., Honjo, T., Yamashiro, T. \& Takano-Yamamoto, T. Terminal differentiation of osteoblasts to osteocytes is accompanied by dramatic changes in the distribution of actin-binding proteins. J. Bone Miner. Res. 19, 471-478 (2004).

53. Galli, C., Passeri, G. \& Macaluso, G. M. Osteocytes and WNT: the mechanical control of bone formation. J. Dent. Res. 89, 331-343 (2010).

54. Santos, A., Bakker, A. D. \& Klein-Nulend, J. The role of osteocytes in bone mechanotransduction. Osteoporos. Int 20, 1027-1031 (2009).

55. Temiyasathit, S. \& Jacobs, C. R. Osteocyte primary cilium and its role in bone mechanotransduction. Ann. N. Y Acad. Sci. 1192, 422-428 (2010). 
56. Yavropoulou, M. P. \& Yovos, J. G. The molecular basis of bone mechanotransduction. J. Musculoskelet. Neuronal Interact. 16, 221-236 (2016).

57. Winslow, M. M. et al. Calcineurin/NFAT signaling in osteoblasts regulates bone mass. Dev. Cell 10, 771-782 (2006).

58. Li, J., Duncan, R. L., Burr, D. B. \& Turner, C. H. L-type calcium channels mediate mechanically induced bone formation in vivo. J. Bone Miner. Res. 17, 1795-1800 (2002).

59. Sun, Z., Costell, M. \& Fassler, R. Integrin activation by talin, kindlin and mechanical forces. Nat. Cell Biol. 21, 25-31 (2019).

60. O'Neill, C. A. \& Galasko, C. S. Calcium mobilization is required for spreading in human osteoblasts. Calcif. Tissue Int. 67, 53-59 (2000).

61. Wei, C. et al. Calcium flickers steer cell migration. Nature 457, 901-905 (2009).

62. Giannone, G., Ronde, P., Gaire, M., Haiech, J. \& Takeda, K. Calcium oscillations trigger focal adhesion disassembly in human U87 astrocytoma cells. J. Biol. Chem 277, 26364-26371 (2002)

63. Giannone, G. et al. Calcium rises locally trigger focal adhesion disassembly and enhance residency of focal adhesion kinase at focal adhesions. J. Biol. Chem. 279, 28715-28723 (2004).

64. Leucht, P., Kim, J. B., Currey, J. A., Brunski, J. \& Helms, J. A. FAK-mediated mechanotransduction in skeletal regeneration. PLoS ONE 2, e390 (2007).

65. Kudryashova, E. et al. Actin cross-linking toxin is a universal inhibitor of tandemorganized and oligomeric G-actin binding proteins. Curr. Biol.: CB 28, 1536-1547 e1539 (2018).

66. Spudich, J. A. \& Watt, S. The regulation of rabbit skeletal muscle contraction. J. Biol. Chem. 246, 4866-4871 (1971).

67. Durer, Z. A. et al. Structural states and dynamics of the D-loop in actin. Biophys. J. 103, 930-939 (2012).

68. Kudryashova, E. et al. Thermodynamic instability of viral proteins is a pathogenassociated molecular pattern targeted by human defensins. Sci. Rep. 6, 32499 (2016).

69. Kudryashova, E. et al. Human defensins facilitate local unfolding of thermodynamically unstable regions of bacterial protein toxins. Immunity 41, 709-721 (2014).

70. Schoenmakers, T. J., Visser, G. J., Flik, G. \& Theuvenet, A. P. CHELATOR: an improved method for computing metal ion concentrations in physiological solutions. Bio. Techn. 12, 870-874 (1992). 876-879.

71. Schindelin, J. et al. Fiji: an open-source platform for biological-image analysis. Nat. Methods 9, 676-682 (2012)

72. Schindelin, J., Rueden, C. T., Hiner, M. C. \& Eliceiri, K. W. The ImageJ ecosystem: an open platform for biomedical image analysis. Mol. Reprod. Dev. 82, 518-529 (2015).

73. Mindell, J. A. \& Grigorieff, N. Accurate determination of local defocus and specimen tilt in electron microscopy. J. Struct. Biol. 142, 334-347 (2003).

74. Tang, G. et al. EMAN2: an extensible image processing suite for electron microscopy. J. Struct. Biol. 157, 38-46 (2007).
75. Scheres, S. H. RELION: implementation of a Bayesian approach to cryo-EM structure determination. J. Struct. Biol. 180, 519-530 (2012).

76. Waterhouse, A. et al. SWISS-MODEL: homology modelling of protein structures and complexes. Nucleic Acids Res. 46, W296-W303 (2018).

77. Pettersen, E. F. et al. UCSF Chimera-a visualization system for exploratory research and analysis. J. Comput. Chem. 25, 1605-1612 (2004).

78. Song, Y. et al. High-resolution comparative modeling with RosettaCM. Structure 21, 1735-1742 (2013).

79. Emsley, P., Lohkamp, B., Scott, W. G. \& Cowtan, K. Features and development of Coot. Acta Crystallogr. Sect. D., Biol. Crystallogr. 66, 486-501 (2010).

80. Phenix, C. P. et al. Imaging of enzyme replacement therapy using PET. Proc. Nat Acad. Sci. USA 107, 10842-10847 (2010).

81. Chen, V. B. et al. MolProbity: all-atom structure validation for macromolecular crystallography. Acta Crystallogr. Sect. D., Biol. Crystallogr. 66, 12-21 (2010).

82. Spatz, J. M. et al. The Wnt inhibitor sclerostin is up-regulated by mechanical unloading in osteocytes in vitro. J. Biol. Chem. 290, 16744-16758 (2015).

83. Bairoch, A. The cellosaurus, a cell-line knowledge resource. J. Biomol. Tech. 29, 25-38 (2018)

84. Uphoff, C. C. \& Drexler, H. G. Detection of mycoplasma contamination in cell cultures. Curr. Protoc. Mol. Biol. 106, 2824 21-14 (2014).

85. Chu, J. et al. Non-invasive intravital imaging of cellular differentiation with a bright red-excitable fluorescent protein. Nat. Methods 11, 572-578 (2014)

86. Kelley, L. A., Mezulis, S., Yates, C. M., Wass, M. N. \& Sternberg, M. J. The Phyre2 web portal for protein modeling, prediction and analysis. Nat. Protoc. 10, 845-858 (2015).

87. Corpet, F. Multiple sequence alignment with hierarchical clustering. Nucleic Acids Res. 16, 10881-10890 (1988).

\section{(i)}

Open Access This article is licensed under a Creative Commons Attribution 4.0 International License, which permits use, sharing, adaptation, distribution and reproduction in any medium or format, as long as you give appropriate credit to the original author(s) and the source, provide a link to the Creative Commons license, and indicate if changes were made. The images or other third party material in this article are included in the article's Creative Commons license, unless indicated otherwise in a credit line to the material. If material is not included in the article's Creative Commons license and your intended use is not permitted by statutory regulation or exceeds the permitted use, you will need to obtain permission directly from the copyright holder. To view a copy of this license, visit http://creativecommons. org/licenses/by/4.0/.

(c) The Author(s) 2020 